\title{
Polynomial bivariate copulas of degree five: characterization and some particular inequalities
}

https://doi.org/10.1515/demo-2021-0101

Received November 21, 2020; accepted February 10, 2021

\begin{abstract}
Bivariate polynomial copulas of degree 5 (containing the family of Eyraud-Farlie-GumbelMorgenstern copulas) are in a one-to-one correspondence to certain real parameter triplets $(a, b, c)$, i.e., to some set of polynomials in two variables of degree 1: $p(x, y)=a x+b y+c$. The set of the parameters yielding a copula is characterized and visualized in detail. Polynomial copulas of degree 5 satisfying particular (in)equalities (symmetry, Schur concavity, positive and negative quadrant dependence, ultramodularity) are discussed and characterized. Then it is shown that for polynomial copulas of degree 5 the values of several dependence parameters (including Spearman's rho, Kendall's tau, Blomqvist's beta, and Gini's gamma) lie in exactly the same intervals as for the Eyraud-Farlie-Gumbel-Morgenstern copulas. Finally we prove that these dependence parameters attain all possible values in ]-1, 1[ if polynomial copulas of arbitrary degree are considered.
\end{abstract}

Keywords: Copula, polynomial inequality, cylindrical algebraic decomposition, dependence parameter, Schur concavity, ultramodularity

MSC: Primary 26B25, 62E10; Secondary 39B62, 60E05, 62H20

\section{Introduction}

In this paper, we will restrict ourselves to two-dimensional (or bivariate) copulas, and we will simply call them copulas. Recall that a copula is a bivariate cumulative distribution function (restricted to $[0,1]^{2}$ ) with uniform marginals on $[0,1]$, which captures the whole dependence structure of the random pair [90].

The name "copula" for functions linking an $n$-dimensional distribution and its one-dimensional marginals goes back to Sklar [90], in which paper he proved a result which is usually referred to as Sklar's theorem. The exact mathematical relationship for $n=2$ is recalled in Theorem 2.1 below. However, links

\footnotetext{
*Corresponding Author: Erich Peter Klement: Department of Knowledge-Based Mathematical Systems, Johannes Kepler University, Linz, Austria, E-mail: ep.klement@jku.at, ORCID 0000-0002-2054-3286

Adam Šeliga: Department of Mathematics and Descriptive Geometry, Faculty of Civil Engineering, Slovak University of Technology, Bratislava, Slovakia, E-mail: adam.seliga@stuba.sk, ORCID 0000-0002-3215-6195

Manuel Kauers: Institute for Algebra, Johannes Kepler University, Linz, Austria, E-mail: manuel.kauers@jku.at, ORCID 00000001-8641-6661

Susanne Saminger-Platz: Department of Knowledge-Based Mathematical Systems, Johannes Kepler University, Linz, Austria, E-mail: susanne.saminger-platz@jku.at, ORCID 0000-0002-9606-5751

Radko Mesiar: Department of Mathematics and Descriptive Geometry, Faculty of Civil Engineering, Slovak University of Technology, Bratislava, Slovakia, E-mail: radko.mesiar@stuba.sk, ORCID 0000-0001-6503-949X

Anna Kolesárová: Institute of Information Engineering, Automation and Mathematics, Faculty of Chemical and Food Technology, Slovak University of Technology, Bratislava, Slovakia, E-mail: anna.kolesarova@stuba.sk, ORCID 0000-0001-6438-0039
} 
between multivariate distributions and their one-dimensional marginals have been studied before, e.g., by Hoeffding [39, 40], Fréchet [36], Dall'Aglio [18-20], and Féron [35], and also later on without any reference to the concept of copulas (see, e.g., [81, 98]).

There are infinitely many copulas: in the books [43, 66] and, more recently, [30] one finds plenty of examples of parametric families (usually with one or two parameters) of copulas and classes of copulas which can be constructed and characterized by functions in one variable (e.g., by additive and/or multiplicative generators $[1,58,82,83]$ in the case of Archimedean copulas).

One of the basic copulas is the independence or product copula $\Pi$ which is a polynomial of degree two in two variables. As a consequence of, e.g., [92], $\Pi$ is the only polynomial copula of degree 2, and there exists no polynomial copula of degree 3 . Polynomial copulas are due to their nature absolutely continuous admitting a representation by their density and discussion of its properties. It is worth noting that quadratic and cubic polynomials have been used in different ways to construct (not necessarily polynomial) copulas, e.g., in [11, 22, 51-54, 67, 71-73, 96, 97, 99, 100] (see also [9, 69, 70]).

The Eyraud-Farlie-Gumbel-Morgenstern copulas (EFGM-copulas) [31, 32, 38, 64] (quite often called FarlieGumbel-Morgenstern copulas) form one of the most remarkable families of copulas. It is not difficult to see that these copulas, which are given by (2.5) below and which are widely used when modeling stochastic dependence, are the only polynomial copulas of degree 4. Unfortunately, all the values of several dependence parameters, as discussed in Definition 6.1, of EFGM-copulas are contained in the interval $\left[-\frac{1}{3}, \frac{1}{3}\right]$ as a consequence of (6.2), so only small dependencies can be modeled. For this reason, several (not necessarily polynomial) extensions and generalizations of EFGM copulas have been considered in the literature (see, e.g., $[3,7,8,17,27,41,42,55,56,62,88,89]$ and the recent survey [78]).

In this contribution, we present a thorough analysis and characterization of polynomial copulas of degree 5 (which are a natural extension of EFGM copulas), and prove some results for the dependence parameters of polynomial copulas of higher degrees.

The paper is organized as follows: after some necessary preliminaries in Section 2, several basic aspects of polynomial copulas of degree 5 are discussed in Section 3. The main results are contained in Section 4 where analytic formulas for the level sets of the parameter set of polynomial copulas of degree 5 are determined with the help of advanced techniques of computer algebra, i.e., by solving a quantifier elimination problem by means of cylindrical algebraic decomposition based on Collins' algorithm [16].

The classification of several subclasses of polynomial copulas of degree 5 satisfying additional properties such as symmetry, Schur concavity [80], ultramodularity [60], and positive and negative quadrant dependence $[57,66]$ are given in Section 5 . In Section 6, several results concerning dependence parameters as given in Definition 6.1 for polynomial copulas of degree 5 and higher are presented.

\section{Preliminaries}

Formally, a (bivariate) copula [90] $C:[0,1]^{2} \rightarrow[0,1]$ satisfies, for each $x \in[0,1]$, the two boundary conditions (C1)

$$
C(x, 0)=C(0, x)=0 \quad \text { and } \quad C(x, 1)=C(1, x)=x,
$$

and it is 2-increasing (C2), i.e., for all $(x, y),\left(x^{\prime}, y^{\prime}\right) \in[0,1]$ with $(x, y) \leq\left(x^{\prime}, y^{\prime}\right)$

$$
C(x, y)+C\left(x^{\prime}, y^{\prime}\right)-C\left(x, y^{\prime}\right)-C\left(x^{\prime}, y\right) \geq 0 .
$$

Sklar's Theorem [90] (see also [30, 44, 66]) states that the link between a bivariate probability distribution and its marginals is necessarily a copula. Alternative ways to prove Sklar's Theorem (also for the case of $n$ dimensional probability distributions) can be found in $[4,25,26,33,34,68,76]$.

Theorem 2.1 (Sklar's Theorem). Let $(\Omega, \mathcal{A}, P)$ be a probability space, let $X, Y: \Omega \rightarrow \mathbb{R}$ be two random variables, and denote the corresponding distribution functions by $F_{X}: \mathbb{R} \rightarrow[0,1]$ and $F_{Y}: \mathbb{R} \rightarrow[0,1]$, respectively. 
(i) There exists a copula $C:[0,1]^{2} \rightarrow[0,1]$ such that, for each $(u, v) \in \mathbb{R}^{2}$, the joint distribution function $F_{X, Y}: \mathbb{R}^{2} \rightarrow[0,1]$ of the random vector $(X, Y)$ is given by

$$
F_{X, Y}(u, v)=C\left(F_{X}(u), F_{Y}(v)\right) .
$$

The copula $C$ is uniquely determined by (2.1) if and only if both random variables $X$ and $Y$ are continuous.

(ii) For each copula $C:[0,1]^{2} \rightarrow[0,1]$ the function $F_{X, Y}: \mathbb{R}^{2} \rightarrow[0,1]$ given by (2.1) is a two-dimensional distribution function.

A copula $C:[0,1]^{2} \rightarrow[0,1]$ is called absolutely continuous if there exists a function $\varphi_{C}:[0,1]^{2} \rightarrow[0, \infty[$ such that for each $(x, y) \in[0,1]^{2}$

$$
C(x, y)=\int_{0}^{y} \int_{0}^{x} \varphi_{C}(u, v) d u d v .
$$

Note that for each $(x, y) \in[0,1]^{2}$ for which the mixed derivative of $C$ below exists we may put

$$
\varphi_{C}(x, y)=\frac{\partial^{2} C(x, y)}{\partial x \partial y} .
$$

The three basic copulas are the Fréchet-Hoeffding lower bound $W:[0,1]^{2} \rightarrow[0,1]$, the product copula $\Pi:[0,1]^{2} \rightarrow[0,1]$ and the Fréchet-Hoeffding upper bound $M:[0,1]^{2} \rightarrow[0,1]$ given by, respectively,

$$
W(x, y)=\max (x+y-1,0), \quad \Pi(x, y)=x y, \quad M(x, y)=\min (x, y) .
$$

Stochastically speaking, random variables $X$ and $Y$ whose copula equals $\Pi$ are called independent, and random variables whose copula equals $M$ or $W$ are called comonotone or countermonotone, respectively.

A particularly interesting and important family of copulas is $\left(C_{\theta}^{\mathbf{E F G M}}\right)_{\theta \in[-1,1]}$ as defined below in (2.5), usually referred to as the family of Farlie-Gumbel-Morgenstern copulas [32, 38, 64, 66]. In [30] (see also [13, $14,63])$ it was pointed out that the corresponding distributions had already been investigated in the earlier and, for many years, forgotten work by Eyraud [31]. In recognition of these early achievements of Eyraud, we will consistently use the name Eyraud-Farlie-Gumbel-Morgenstern copulas (EFGM copulas for short) in this paper. This family of copulas is used quite often when the weak dependence of exchangeable random variables is modeled.

For each parameter $\theta \in[-1,1]$ the Eyraud-Farlie-Gumbel-Morgenstern copula $C_{\theta}^{\mathrm{EFGM}}:[0,1]^{2} \rightarrow[0,1]$ is defined by

$$
C_{\theta}^{\mathbf{E F G M}}(x, y)=x y+\theta x y(1-x)(1-y),
$$

and since the mixed derivative $\frac{\partial^{2} C_{\theta}^{\mathrm{EGGM}}}{\partial x \partial y}$ exists and is non-negative for each $(x, y) \in[0,1]^{2}$, the corresponding density $\varphi_{C_{\theta}^{\mathrm{EFGM}}}:[0,1]^{2} \rightarrow[0, \infty[$ is given by

$$
\varphi_{C_{\theta}^{\mathrm{EFGM}}}(x, y)=\frac{\partial^{2} C_{\theta}^{\mathrm{EFGM}}}{\partial x \partial y}(x, y)=1+\theta(1-2 x)(1-2 y) .
$$

Obviously, we have $\Pi=C_{0}^{\mathrm{EFGM}}$. The copulas $W$ and $M$, however, do not belong to the family of EFGM copulas.

For more information concerning copulas including examples, constructions and applications, we refer to the monographs $[2,30,43,44,66,84]$.

The Eyraud-Farlie-Gumbel-Morgenstern copulas $\left(C_{\theta}^{\mathbf{E F G M}}\right)_{\theta \in[-1,1]}$ have some nice properties: being defined by polynomials (of degree 4), they can be easily computed, and they are absolutely continuous. But the EFGM copulas also have some limitations: under identically distributed marginals they can only generate an exchangeable random pair. Also, the possible values of some well-known dependence parameters are contained in some rather small subintervals of $[-1,1]$ (in $\left[-\frac{1}{3}, \frac{1}{3}\right]$ for Spearman's rho, in $\left[-\frac{2}{9}, \frac{2}{9}\right]$ for Kendall's tau, in $\left[-\frac{1}{4}, \frac{1}{4}\right]$ for Blomqvist's beta, and in $\left[-\frac{4}{15}, \frac{4}{15}\right]$ for Gini's gamma, i.e., only weak dependencies can be modeled), and there are linear links between these dependence parameters. We shall have a closer look at dependence parameters of the Eyraud-Farlie-Gumbel-Morgenstern copulas and of polynomial copulas in Section 6. 
To keep the advantages and to overcome (some of) the limitations mentioned above, one can consider polynomial copulas of higher degrees (this idea was mentioned but not further developed in [92]). In this paper we shall study copulas defined by polynomials in two variables and call them polynomial copulas.

For $n \in \mathbb{N}_{0}=\mathbb{N} \cup\{0\}$ consider the polynomial $p: \mathbb{R}^{2} \rightarrow \mathbb{R}$ in two variables of degree $n$ given by

$$
p(x, y)=\sum_{k=0}^{n} \sum_{i=0}^{k} a_{k, i} x^{k-i} y^{i},
$$

with coefficients $a_{0,0}, a_{1,0}, a_{0,1}, \ldots, a_{n, 0}, a_{n, 1}, \ldots, a_{n, n} \in \mathbb{R}$.

If $a_{n, 0}=a_{n, 1}=\cdots=a_{n, n}=0$ then $p$ is called an improper polynomial of degree $n$, and a proper polynomial of degree $n$ otherwise. For each improper polynomial $p$ of degree $n$ (with the only exception when all coefficients equal 0 - the zero polynomial 0 has no universally accepted degree: sometimes it is said to be undefined, sometimes it is defined as $\operatorname{deg}(0)=-1$ or as $\operatorname{deg}(0)=-\infty)$ there is a number $m \in\{0,1, \ldots, n-1\}$ such that $p$ is a proper polynomial of degree $m$.

Definition 2.2. A bivariate copula $C:[0,1]^{2} \rightarrow[0,1]$ is called a polynomial copula if the function $C$ is a polynomial in two variables.

Example 2.3. In the literature one can find several examples of polynomial copulas. We only mention a few of them:

(i) Each EFGM copula $C_{\theta}^{\mathrm{EFGM}}$ given by (2.5) is a polynomial copula of degree 4, and only $\Pi=C_{0}^{\mathrm{EFGM}}$ is an improper polynomial copula of degree 4 (in fact, it is a proper polynomial copula of degree 2).

(ii) From $\left[53\right.$, Theorem 1] (see also $[23,47,52]$ ) we know that, for each copula $C:[0,1]^{2} \rightarrow[0,1]$, also the function $D_{C}:[0,1]^{2} \rightarrow[0,1]$ given by

$$
D_{C}(x, y)=C(x, y)(x+y-C(x, y))
$$

is a copula. Starting with an arbitrary polynomial copula $C$ of degree $k$, we can use (2.6) to construct a sequence of polynomial copulas $\left(C^{(n)}\right)_{n \in \mathbb{N}_{0}}$ inductively by

$$
C^{(n)}= \begin{cases}C & \text { if } n=0 \\ D_{C^{(n-1)}} & \text { if } n \geq 1\end{cases}
$$

Clearly, for each $n \in \mathbb{N}_{0}$ the polynomial copula $C^{(n)}$ is of degree $2^{n} \cdot k$. In particular, if $C=\Pi$ or $C=C_{\theta}^{\mathrm{EFGM}}$ with $\theta \neq 0$ then, for each $n \in \mathbb{N}_{0}$, the polynomial copula $\Pi^{(n)}$ constructed via (2.7) is of degree $2^{n+1}$, and the polynomial copula $C_{\theta}^{\mathbf{E F G M}}{ }^{(n)}$ with $\theta \neq 0$ is of degree $2^{n+2}$.

(iii) From [59] we know that the function $C:[0,1]^{2} \rightarrow[0,1]$ given by

$$
C(x, y)=\frac{1}{2} x y\left(1+(x+y-x y)^{2}\right)
$$

is a polynomial copula of degree 6 which cannot be obtained using construction (2.7), but it can be (compare (2.9) below) rewritten as

$$
C(x, y)=x y+\frac{1}{2}(x y-x-y-1) x(1-x) y(1-y) .
$$

In [92] it was shown that a copula $C:[0,1]^{2} \rightarrow[0,1]$ is a polynomial copula only if for all $(x, y) \in[0,1]^{2}$

$$
C(x, y)=x y+p(x, y) x(1-x) y(1-y),
$$

where $p: \mathbb{R}^{2} \rightarrow \mathbb{R}$ is some appropriate polynomial in two variables. It is obvious that not for each polynomial $p$ in two variables the function $C$ given by (2.9) is a copula: trivial counterexamples are the constant polynomials $p(x, y)=\theta$ with $\theta \notin[-1,1]$ which are not 2-increasing, i.e., violate (C2).

Remark 2.4. Let $C:[0,1]^{2} \rightarrow[0,1]$ be a polynomial copula given by (2.9). 
(i) If $C \neq \Pi$ then $\operatorname{deg}(C)=\operatorname{deg}(p)+4$ and $\operatorname{deg}\left(\varphi_{C}\right)=\operatorname{deg}(C)-2$, where $\varphi_{C}:[0,1]^{2} \rightarrow[0, \infty[$ is the density of $C$ given by (2.3).

(ii) If $p$ equals the zero polynomial then the corresponding polynomial copula $C$ (of degree 2) equals $\Pi$, the product copula.

(iii) Obviously, there is no proper polynomial copula $C$ with $\operatorname{deg}(C)=3$.

(iv) If $p$ is a constant polynomial, i.e., $p(x, y)=\theta$ for some $\theta \in \mathbb{R}$ then $C$ is a polynomial copula of degree 4 if and only if $\theta \in[-1,1]$, i.e., $C=C_{\theta}^{\mathrm{EFGM}}$.

(v) Generally, if $C$ is a proper polynomial copula of degree $n \geq 4$ then the polynomial $p$ has $\frac{(n-2)(n-3)}{2}$ coefficients.

It is evident that any polynomial function $C:[0,1]^{2} \rightarrow[0,1]$ which satisfies the boundary conditions (C1) of copulas is necessarily of the form (2.9), i.e.,

$$
C(x, y)=x y+p(x, y) x(1-x) y(1-y) .
$$

For each polynomial $p: \mathbb{R}^{2} \rightarrow \mathbb{R}$, this function is absolutely continuous, the mixed derivative $\frac{\partial^{2} C(x, y)}{\partial x \partial y}$ exists for each $(x, y) \in[0,1]^{2}$, and the density $\varphi_{C}:[0,1]^{2} \rightarrow \mathbb{R}$ is given by

$$
\begin{aligned}
\varphi_{C}(x, y)= & 1+\frac{\partial^{2} p(x, y)}{\partial x \partial y}\left(x-x^{2}\right)\left(y-y^{2}\right)+\frac{\partial p(x, y)}{\partial x}\left(x-x^{2}\right)(1-2 y) \\
& +\frac{\partial p(x, y)}{\partial y}(1-2 x)\left(y-y^{2}\right)+p(x, y)(1-2 x)(1-2 y) .
\end{aligned}
$$

Then $C$ satisfies (C2), i.e., it is 2-increasing and, therefore, a copula, if and only if $\varphi_{C}(x, y) \geq 0$ for each $(x, y) \in$ $[0,1]^{2}$, i.e., $C$ has a non-negative density.

Summarizing all the facts which we have mentioned so far, we have obtained the following result:

Proposition 2.5. A function $C:[0,1]^{2} \rightarrow[0,1]$ is a polynomial copula if and only if there exists a polynomial $p: \mathbb{R}^{2} \rightarrow \mathbb{R}$ in two variables such that $C$ is given by (2.9), i.e., $C(x, y)=x y+p(x, y) x(1-x) y(1-y)$, and the density $\varphi_{C}:[0,1]^{2} \rightarrow \mathbb{R}$ given by (2.10) is non-negative, i.e., $\frac{\partial^{2} C(x, y)}{\partial x \partial y} \geq 0$ for all $(x, y) \in[0,1]^{2}$.

In the monograph [24], where some results from [75] are cited, the following (which has been cited in [65, Subsection 4.4]) is claimed to be an alternative formula for polynomial copulas $C:[0,1]^{2} \rightarrow[0,1]$ of degree $n$ :

$$
\left.C(x, y)=x y\left(1+\sum_{j \geq 1, k \geq 1, j+k \leq n-2} \frac{\theta_{j, k}}{(j+1)(k+1)}\left(x^{j}-1\right)\left(y^{k}-1\right)\right)\right),
$$

subject to the condition

$$
0 \leq \min \left(\sum_{j \geq 1, k \geq 1, j+k \leq n-2} \frac{k \theta_{j, k}}{(j+1)(k+1)}, \sum_{j \geq 1, k \geq 1, j+k \leq n-2} \frac{j \theta_{j, k}}{(j+1)(k+1)}\right) \leq 1 .
$$

It is easy to see that formulas (2.11) and (2.9) (as given in [92]) are identical. As we shall see in Example 4.1 below, in general the condition (2.12) is neither sufficient nor necessary for $C$ given by (2.11) being a copula.

The characterization of the set of polynomials $p$ of degree $n \in \mathbb{N}$ such that the function given by (2.9) is a (proper) polynomial copula of degree 5 or higher is not trivial at all.

The simplest case is to consider polynomials $p: \mathbb{R}^{2} \rightarrow \mathbb{R}$ of degree 1, i.e., $p(x, y)=a x+b y+c$ for some $a, b, c \in \mathbb{R}$. In this case we have to determine the set of parameters $(a, b, c) \in \mathbb{R}^{3}$ such that $C_{(a, b, c)}:[0,1]^{2} \rightarrow$ $[0,1]$ given by

$$
C_{(a, b, c)}(x, y)=x y+(a x+b y+c) x(1-x) y(1-y)
$$

is a copula.

In this paper we give a complete characterization and 3D illustrations of the set of parameters $(a, b, c) \in$ $\mathbb{R}^{3}$ such that formula (2.13) yields a copula. In Section 5, possible additional properties of polynomial copulas 
of degree 5 such as symmetry, Schur concavity, ultramodularity, and both positive and negative quadrant dependence are discussed. Section 6 is devoted to dependence parameters (including Spearman's rho, Kendall's tau, Blomqvist's beta, and Gini's gamma) for polynomial copulas of degree 5 (where they behave in a similar way as for the EFGM copulas) and for polynomial copulas with arbitrary degree (where all possible values in the interval ] $-1,1[$ are attained).

\section{Polynomial copulas of degree five}

Our focus is the study of polynomial copulas of degree 5. It is evident that any polynomial function $C:[0,1]^{2} \rightarrow[0,1]$ of degree 5 which satisfies the boundary conditions (C1) of copulas is necessarily of the form (2.13), i.e., $C=C_{(a, b, c)}$ for some triplet $(a, b, c) \in \mathbb{R}^{3}$. Obviously, each $C_{(a, b, c)}$ with $(a, b, c) \in \mathbb{R}^{3}$ is absolutely continuous, the mixed derivative $\frac{\partial^{2} C_{(a, b, c)}(x, y)}{\partial x \partial y}$ exists for each $(x, y) \in[0,1]^{2}$, and the density $\varphi_{C_{(a, b, c)}}:[0,1]^{2} \rightarrow \mathbb{R}$ is given by

$$
\varphi_{C_{(a, b, c)}}(x, y)=1+a\left(x-x^{2}\right)(1-2 y)+b(1-2 x)\left(y-y^{2}\right)+(a x+b y+c)(1-2 x)(1-2 y) .
$$

Then $C_{(a, b, c)}$ satisfies (C2), i.e., it is 2-increasing and, therefore, a copula, if and only if $\varphi_{C_{(a, b, c)}}(x, y) \geq 0$ for each $(x, y) \in[0,1]^{2}$, i.e., $C_{(a, b, c)}$ has a non-negative density.

As an immediate consequence of Proposition 2.5 we obtain the following characterization of polynomial copulas of degree 5 .

Corollary 3.1. A function $C:[0,1]^{2} \rightarrow[0,1]$ is a polynomial copula of degree 5 if and only if there exists a triplet $(a, b, c) \in \mathbb{R}^{3}$ such that $C=C_{(a, b, c)}$, where $C_{(a, b, c)}:[0,1]^{2} \rightarrow[0,1]$ is given by (2.13), i.e., $C(x, y)=$ $C_{(a, b, c)}(x, y)=x y+(a x+b y+c) x(1-x) y(1-y)$, and the density $\varphi_{C_{(a, b, c)}}:[0,1]^{2} \rightarrow \mathbb{R}$ given by (3.1) is nonnegative, i.e., for all $(x, y) \in[0,1]^{2}$

$$
1+a\left(x-x^{2}\right)(1-2 y)+b(1-2 x)\left(y-y^{2}\right)+(a x+b y+c)(1-2 x)(1-2 y) \geq 0 .
$$

From [30, Theorem 1.7.5] we know that the limit of each pointwise convergent sequence of copulas is also a copula. In particular, a sequence $\left(C_{\left(a_{n}, b_{n}, c_{n}\right)}\right)_{n \in \mathbb{N}}$ of polynomial copulas of degree 5 converges if and only if the three sequences $\left(a_{n}\right)_{n \in \mathbb{N}},\left(b_{n}\right)_{n \in \mathbb{N}}$, and $\left(c_{n}\right)_{n \in \mathbb{N}}$ converge to some numbers $a, b$ and $c$, respectively. As a consequence, for a converging sequence $\left(C_{\left(a_{n}, b_{n}, c_{n}\right)}\right)_{n \in \mathbb{N}}$ of polynomial copulas of degree 5 we have

$$
\lim _{n \rightarrow \infty} C_{\left(a_{n}, b_{n}, c_{n}\right)}=C_{\left(\lim _{n \rightarrow \infty} a_{n}, \lim _{n \rightarrow \infty} b_{n}, \lim _{n \rightarrow \infty} c_{n}\right)},
$$

showing that the set of polynomial copulas of degree 5 is closed.

The polynomial copulas of degree 5 provide a natural extension of the family of Eyraud-Farlie-GumbelMorgenstern copulas $\left(C_{\theta}^{\mathbf{E F G M}}\right)_{\theta \in[-1,1]}$ given by (2.5): a triplet $(0,0, c) \in \mathbb{R}^{3}$ (i.e., if we put $a=b=0$ ) satisfies (3.2) for all $(x, y) \in[0,1]^{2}$ if and only if $c \in[-1,1]$, and the family of copulas $\left(C_{(0,0, c)}\right)_{c \in[-1,1]}$ coincides with the family of EFGM copulas (which are the only improper polynomial copulas of degree 5). Other extensions and generalizations of the family of EFGM copulas are presented and discussed in $[3,17,41,42,55,56$, 62].

\section{Level sets of the parameter set PC5}

We shall denote the set of all triplets $(a, b, c) \in \mathbb{R}^{3}$ turning the function $C_{(a, b, c)}:[0,1]^{2} \rightarrow[0,1]$ given by (2.13) into a (bivariate) copula by PC5. According to Corollary 3.1 we have

$$
\text { PC5 }=\left\{(a, b, c) \in \mathbb{R}^{3} \mid(3.2) \text { holds for each }(x, y) \in[0,1]^{2}\right\} .
$$




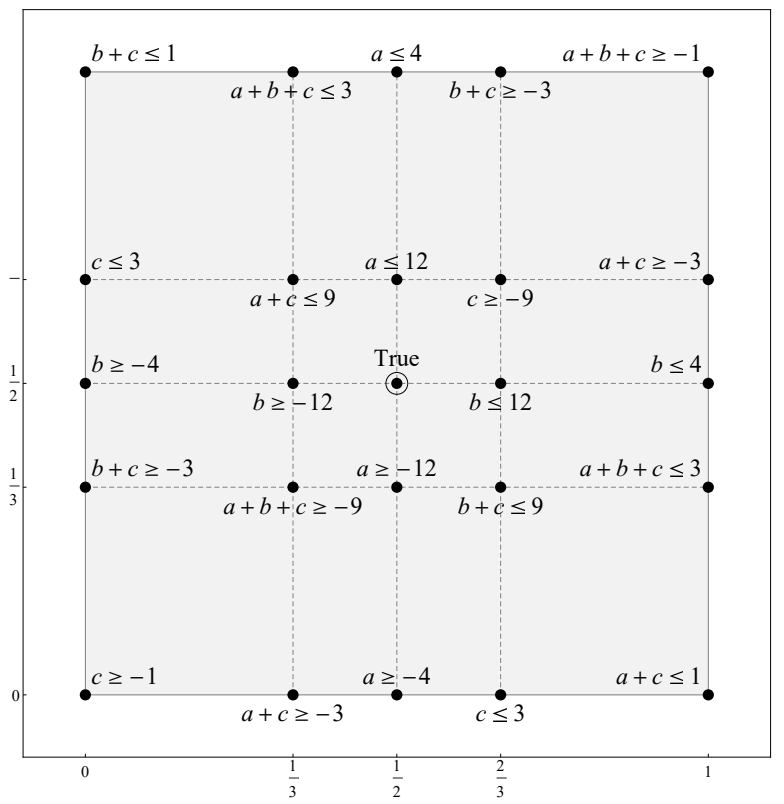

Figure 1: Each of the 24 linear inequalities is obtained when inserting the coordinates of the point in $\left\{0, \frac{1}{3}, \frac{1}{2}, \frac{2}{3}, 1\right\}^{2}$ next to it into (4.3). The special role of $\left(\frac{1}{2}, \frac{1}{2}\right)$ where $\varphi_{C_{(a, b, c)}}\left(\frac{1}{2}, \frac{1}{2}\right)=1$ is indicated by "True".

The convexity of both the set of all bivariate copulas and the set of all polynomials of degree 1 implies the convexity of the parameter set PC5.

It is obvious that for each triplet $(a, b, c) \in \mathbb{R}^{3}$ the assertions " $(a, b, c) \in \mathbf{P C 5}$ ” and “ $(b, a, c) \in \mathbf{P C} 5$ ” are equivalent. This means that the parameter set PC5 is symmetric with respect to the plane determined by $a-b=0$, i.e., PC5 is invariant under reflections through the plane $a-b=0$. We shall denote the intersection of PC5 with the plane $a-b=0$ by PC5 sym $_{\text {(see Figure 7), i.e., }}$

$$
\mathbf{P C 5}_{\text {sym }}=\mathbf{P C 5} \cap\left\{(a, b, c) \in \mathbb{R}^{3} \mid a=b\right\} .
$$

Note that $(a, b, c) \in \mathbf{P C} 5_{\text {sym }}$ if and only if the corresponding copula $C_{(a, b, c)}$ is symmetric and Schur concave (see Corollary 5.1 and Proposition 5.2). Another consequence is that several dependence parameters (see Section 6) coincide for the copulas $C_{(a, b, c)}$ and $C_{(b, a, c)}$.

For each $\zeta \in \mathbb{R}$ the level set PC5 $5^{[\zeta]}$ of the parameter set PC5 is defined by

$$
\mathbf{P C 5}^{[\zeta]}=\{(a, b, c) \in \mathbf{P C 5} \mid c=\zeta\} .
$$

From the convexity of PC5 it readily follows that each level set $\mathbf{P C} 5^{[\zeta]}$ is also a convex subset of $\mathbb{R}^{3}$.

In this section we shall give full analytical descriptions of all level sets PC5 ${ }^{[\zeta]}$ of the parameter set PC5 of the polynomial copulas of degree 5 . These formulas were obtained with the help of the computer algebra software Mathematica ${ }^{\circledR}$.

Note first that we can rewrite the density $\varphi_{C_{(a, b, c)}}$ in a way that the inequality (3.2) looks as follows:

$$
1+c(-1+2 x)(-1+2 y)+a x(-2+3 x)(-1+2 y)+b(-1+2 x) y(-2+3 y) \geq 0 .
$$

We see immediately that the summands of the density $\varphi_{C_{(a, b, c)}}$ change their sign whenever $x, y \in\left\{\frac{1}{2}\right.$, $\left.\frac{2}{3}\right\}$, and that the monotonicity of the density changes whenever $x=\frac{1}{3}$ or $y=\frac{1}{3}$.

If we insert the 25 possible pairs $(x, y) \in\left\{0, \frac{1}{3}, \frac{1}{2}, \frac{2}{3}, 1\right\}^{2}$ into (4.3), it follows that $\varphi_{C_{(a, b, c)}}\left(\frac{1}{2}, \frac{1}{2}\right)=1$ for each $(a, b, c) \in \mathbb{R}^{3}$, i.e., in the point $\left(\frac{1}{2}, \frac{1}{2}\right)$ the density is non-negative for each triplet $(a, b, c) \in \mathbb{R}^{3}$.

For the remaining 24 points in $\left\{0, \frac{1}{3}, \frac{1}{2}, \frac{2}{3}, 1\right\}^{2} \backslash\left\{\left(\frac{1}{2}, \frac{1}{2}\right)\right\}$ we obtain 24 necessary conditions for a nonnegative density $\varphi_{C_{(a, b, c)}}$ which are given in Figure 1. 
Obviously, the 24 inequalities in Figure 1 are highly redundant, and we can reduce them to the following equivalent system of necessary conditions:

$$
\begin{aligned}
& -4 \leq a \leq 4, \quad-3 \leq a+c \leq 1, \quad-1 \leq a+b+c \leq 3, \\
& -4 \leq b \leq 4, \quad-3 \leq b+c \leq 1, \quad-1 \leq c \leq 3 .
\end{aligned}
$$

Using, for an arbitrary subset $A$ of $\mathbb{R}^{n}$, the notation $\operatorname{Conv}(A)$ for the convex hull of $A$, i.e., the set of all convex combinations of finitely many points in $A$ given by

$$
\operatorname{Conv}(A)=\left\{\sum_{i=1}^{k} \lambda_{i} a_{i} \mid a_{1}, \ldots, a_{k} \in A, \lambda_{1}, \ldots, \lambda_{k} \in[0,1] \text { and } \sum_{i=1}^{k} \lambda_{i}=1\right\},
$$

and solving the system of linear inequalities (4.4) we obtain the polyhedron $\mathbf{P}_{\left(B_{1}, B_{2}, B_{3}, B_{4}\right)}$ determined by the four points $B_{1}=(-2,-2,3), B_{2}=(-2,2,-1), B_{3}=(2,-2,-1)$ and $B_{4}=(2,2,-1)$, i.e.,

$$
\mathbf{P}_{\left(B_{1}, B_{2}, B_{3}, B_{4}\right)}=\operatorname{Conv}(\{(-2,-2,3),(-2,2,-1),(2,-2,-1),(2,2,-1)\}) .
$$

An equivalent formulation is that a triplet $(a, b, c) \in \mathbf{P C 5}$ necessarily has to be a solution of the system of linear inequalities

$$
a+c \leq 1, \quad b+c \leq 1, \quad a+b+c \geq-1, \quad c \geq-1 .
$$

Actually, $\mathbf{P}_{\left(B_{1}, B_{2}, B_{3}, B_{4}\right)}$ is a pyramid with apex $B_{1}$ whose base is the triangle determined by the vertices $B_{2}-B_{4}$, and PC5 has to be a subset of $\mathbf{P}_{\left(B_{1}, B_{2}, B_{3}, B_{4}\right)}$, i.e.,

$$
\mathbf{P C 5} \subseteq \mathbf{P}_{\left(B_{1}, B_{2}, B_{3}, B_{4}\right)} .
$$

Since $\mathbf{P}_{\left(B_{1}, B_{2}, B_{3}, B_{4}\right)}$ is a bounded subset of $\mathbb{R}^{3}$, (3.3) and (4.7) imply that PC5 and all level sets PC5 ${ }^{[\zeta]}$ of PC5, as given in (4.2), are compact subsets of $\mathbb{R}^{3}$.

A quick check shows that none of the four vertices $B_{1}, B_{2}, B_{3}, B_{4}$ of the polyhedron $\mathbf{P}_{\left(B_{1}, B_{2}, B_{3}, B_{4}\right)}$ is an element of PC5 because of

$$
\varphi_{C_{(-2,-2,3)}}\left(0, \frac{5}{6}\right)=\varphi_{C_{(-2,2,-1)}}\left(1, \frac{5}{6}\right)=\varphi_{C_{(2,-2,-1)}}\left(0, \frac{1}{6}\right)=\varphi_{C_{(2,2,-1)}}\left(1, \frac{1}{6}\right)=-\frac{1}{6}<0 .
$$

This means that the conditions (4.6) are only necessary but not sufficient, and that the subset relation in (4.7) must be strict. As can be seen from Figure 4, $\mathbf{P}_{\left(B_{1}, B_{2}, B_{3}, B_{4}\right)}$ is the smallest polyhedron containing PC5 as a subset.

Example 4.1. Recall the formula of a polynomial copula of degree $n$ given by (2.11) subject to condition (2.12).

(i) For $n=4$ formula (2.11) and condition (2.12) reduce to

$$
\begin{gathered}
\left.C(x, y)=x y\left(1+\frac{\theta_{1,1}}{4}(x-1)(y-1)\right)\right), \\
0 \leq \frac{\theta_{1,1}}{4} \leq 1 .
\end{gathered}
$$

If we put $\theta=\frac{\theta_{1,1}}{4}$ then (4.8) coincides with the formula for Eyraud-Farlie-Gumbel-Morgenstern copulas given in (2.5). However, for $\frac{\theta_{1,1}}{4} \in[-1,0$ [ condition (4.9) is violated, but formula (4.8) yields a EFGM copula with parameter $\theta \in[-1,0$ [ which is a polynomial copula of degree 4 .

(ii) For $n=5$ formula (2.11) and condition (2.12) reduce to

$$
\begin{gathered}
C(x, y)=x y\left(1+\frac{\theta_{1,1}}{4}(x-1)(y-1)+\frac{\theta_{1,2}}{6}(x-1)\left(y^{2}-1\right)+\frac{\theta_{2,1}}{6}\left(x^{2}-1\right)(y-1)\right), \\
0 \leq \min \left(\frac{\theta_{1,1}}{4}+\frac{\theta_{2,1}}{6}+\frac{2 \theta_{1,2}}{6}, \frac{\theta_{1,1}}{4}+\frac{2 \theta_{2,1}}{6}+\frac{\theta_{1,2}}{6}\right) \leq 1 .
\end{gathered}
$$

If we put $\theta_{1,1}=-4(a+b-c), \theta_{1,2}=6 b$ and $\theta_{2,1}=6 a$ then (4.10) coincides with the formula for $C_{(a, b, c)}$ given in (2.13). 
However, condition (4.11) is neither necessary nor sufficient for $C$ given by (4.10) being a polynomial copula of degree 5. The triplet $(a, b, c)=(0,0,-1)$ belongs to PC5 but the corresponding triplet $\left(\theta_{1,1}, \theta_{1,2}, \theta_{2,1}\right)=(-4,0,0)$ violates condition (4.11). On the other hand, the coordinates $(-2,-2,3)$ of the vertex $B_{1}$ of the pyramid $\mathbf{P}_{\left(B_{1}, B_{2}, B_{3}, B_{4}\right)}$ do not belong to PC5, but the corresponding triplet $\left(\theta_{1,1}, \theta_{1,2}, \theta_{2,1}\right)=(28,-12,-12)$ satisfies condition (4.11).

\subsection{Quantifier Elimination}

Recall that the task of identifying all polynomial copulas of degree 5 consists in determining all real numbers $a, b, c$ such that the inequality (3.2) holds for all $(x, y) \in[0,1]^{2}$, subject to the linear constraints (4.6). In terms of formal logic, these requirements can be phrased as a quantifier elimination problem: we have a formula $\Phi$ consisting of polynomial equations and inequalities in the variables $x, y, a, b, c$, and we are interested in a formula $\Psi$ consisting of polynomial equations and inequalities in the variables $a, b, c$ such that $\Psi$ is equivalent to $\forall x, y: \Phi$ as a statement about the real numbers.

Such quantifier elimination problems can be solved using computer algebra. It was first shown by A. Tarski [95] that the problem is decidable, but the algorithm he proposed is impractical and only of theoretical interest. G. E. Collins [16] has later proposed a more efficient (though still computationally very expensive) algorithm, which is implemented in Mathematica and elsewhere [12, 85, 94]. This technique is extremely powerful and deserves to be better known.

In principle, our quantifier elimination problem can be solved by the following single Mathematica command:

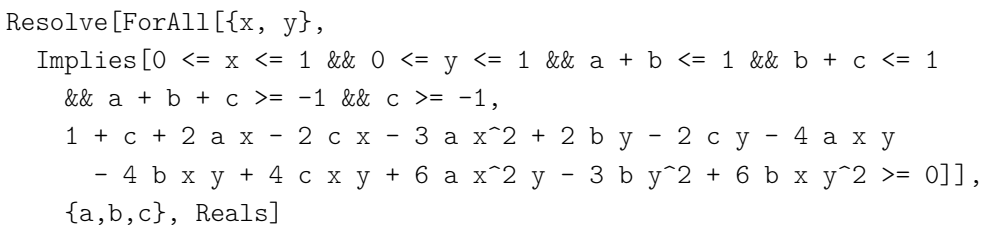

It would even be possible to leave out the linear constraints (4.6), the solution set will be PC5 in either case. Unfortunately however, in neither of the two variants does the computation come to an end within a reasonable amount of time. In order to solve the problem, we need to employ a more low-level command. In order to explain our computation, we first need to give a bit more detail on Collins' algorithm. For further details, see $[6,15,45]$.

Collins' algorithm takes as input a system of polynomial equations and/or inequalities in one or more variables with rational coefficients and returns a logical formula with a very particular structure that describes the solution set of the input system.

For example, applied to the single input inequality $x^{2}+y^{2}-1 \leq 0$, the algorithm as implemented in the Mathematica command CylindricalDecomposition [93] will produce something like this:

$$
(x=-1 \wedge y=0) \vee\left(-1<x<1 \wedge-\sqrt{1-x^{2}} \leq y \leq \sqrt{1-x^{2}}\right) \vee(x=1 \wedge y=0) .
$$

This looks more complicated at first glance, but the structure of the formula has the useful feature that it makes quantifier elimination simple. For example, if we want to know all real numbers $x$ such that $\exists y$ : $x^{2}+y^{2}-1 \leq 0$, all we need to do is to replace all the equations and inequalities in the above formula involving $y$ by True and simplify the result. This will give $x=-1 \vee(-1<x<1) \vee x=1$.

The structure of the output formula can also be used to deal with formulas involving $\forall$, but it is slightly more difficult to explain precisely how it works, and it is not necessary to do so because we can always use $\forall y: \Phi \Longleftrightarrow \neg \exists y: \neg \Phi$ to reduce the task to an existential quantifier.

Almost the entire computation time of Collins' algorithm is spent on determining truth values of formulas in areas of the solution set which are specified by equations that were introduced during the computation. For this reason, the alternative command GenericCylindricalDecomposition is provided by Mathematica. 
It performs Collins' algorithm without paying attention to what happens for the solutions of these equations. For example, applied to $x^{2}+y^{2}-1 \geq 0$, this command produces the pair

$$
\left(x<-1 \vee-1<x<1 \wedge\left(y<-\sqrt{1-x^{2}} \vee y>\sqrt{1-x^{2}}\right) \vee x>1, x-1=0 \vee x+1=0 \vee-1+x^{2}+y^{2}=0\right)
$$

as output. This is to be read as follows: "I have not investigated any points $(x, y) \in \mathbb{R}^{2}$ with $x-1=0$ or $x+1=0$ or $-1+x^{2}+y^{2}=0$, but for all other points, I guarantee that $x^{2}+y^{2}-1 \geq 0$ is true if and only if $x<-1 \vee-1<x<1 \wedge\left(y<-\sqrt{1-x^{2}} \vee y>\sqrt{1-x^{2}}\right) \vee x>1$ is true."

The first part of the output again has a format that makes quantifier elimination simple, but the effect of ignoring a lower-dimensional algebraic set has to be properly taken into account. In the example above, we are entitled to conclude that for almost all real numbers $x$, the formula $x<-1 \vee x>1$ is equivalent to the formula $\forall^{\sim} y: x^{2}+y^{2}-1 \geq 0$, where $\forall^{\sim}$ also means "for almost all".

Let us now return to the polynomial inequality (3.2) and the linear constraints (4.6). While we were not able to solve the quantifier elimination problem using Resolve or CylindricalDecomposition, it turns out that GenericCylindricalDecomposition does the job. Note that this is sufficient for our purpose, because PC5 is a convex and, as a consequence of (3.3) and (4.7), also a compact subset of $\mathbb{R}^{3}$. Therefore, any solution on some hypersurface passing through the solution set can be seen as the limit of a sequence and/or the convex combination of parameter tuples fulfilling the conditions of the guaranteed solution part of the algorithm. Moreover, due to the continuity of copulas there is no harm in using the quantifier $\forall^{\sim}$ instead of $\forall$ on $x$ and $y$.

Since we prefer to eliminate existential quantifiers, we bring the problem $\forall x, y: \Psi(x, y, a, b, c) \Rightarrow$ $\Xi(x, y, a, b, c)$ into the form $\neg \exists x, y: \Psi(x, y, a, b, c) \wedge \neg \Xi(x, y, a, b, c)$. Here, $\Psi$ contains the requirement that $x, y$ are restricted to $[0,1]$ and $a, b, c$ satisfy the inequalities (4.6), and $\Xi$ is (3.2). Applying the command GenericCylindricalDecomposition to the inequalities

$$
\begin{aligned}
0 \leq x \leq 1 \wedge 0 & \leq y \leq 1 \wedge a+b \leq 1 \wedge b+c \leq 1 \wedge a+b+c \geq-1 \wedge c \geq-1 \\
& \wedge 1+c+2 a x-2 c x-3 a x^{2}+2 b y-2 c y-4 a x y-4 b x y+4 c x y+6 a x^{2} y-3 b y^{2}+6 b x y^{2}<0
\end{aligned}
$$

terminates within a few minutes. Depending on the order in which the variables $a, b, c$ are specified, the computation time on a Linux machine with 3GHz CPU and 700Gb RAM ranges between 98 and 251 seconds. The computation also terminates when the inequalities of (4.6) are not taken into account, but the runtime then ranges between 4343 and 14856 seconds. In both variants, the output is extremely large, so that it was impossible to work out the quantifier elimination step by hand, but it is easy to let Mathematica replace all inequalities involving $x$ or $y$ by True. The instructions we used to this end are given in the appendix. After quantifier elimination, the formula is smaller, but still very large. One reason is that the formula produced by GenericCylindricalDecomposition uses False as truth value for all points on the disregarded hypersurface. As a consequence, it contains many subformulas of the form $u<a<v \vee v<a<w$ which, due to the compactness and convexity of the solution set, we can safely simplify to $u<a<w$. The code we used for this simplification is also given in the appendix.

At this point, we have a formula $\Psi$ in $a, b, c$ which is equivalent (possibly up to a set of measure zero) to $\exists^{\sim} x, y: \Psi(x, y, a, b, c) \wedge \neg \Xi(x, y, a, b, c)$, where $\exists^{\sim}$ means that the set of points $(x, y) \in \mathbb{R}^{2}$ with the required property has positive measure. It remains to perform the negation. For getting a more readable result, we call GenericCylindricalDecomposition on the conjunction of (4.6) and $\neg \Psi$ and apply our simplifying routine to the output.

\subsection{Results}

We now present the result of the computations described above.

For each $c \in \mathbb{R}$ the level set PC5 ${ }^{[c]}$ of the parameter set PC5 (which is necessarily a convex set) is fully described by giving the following information for the triplets $(a, b, c) \in \mathbf{P C} 5^{[c]}$ :

(i) the range of $c$ in each of the five cases to be considered, 


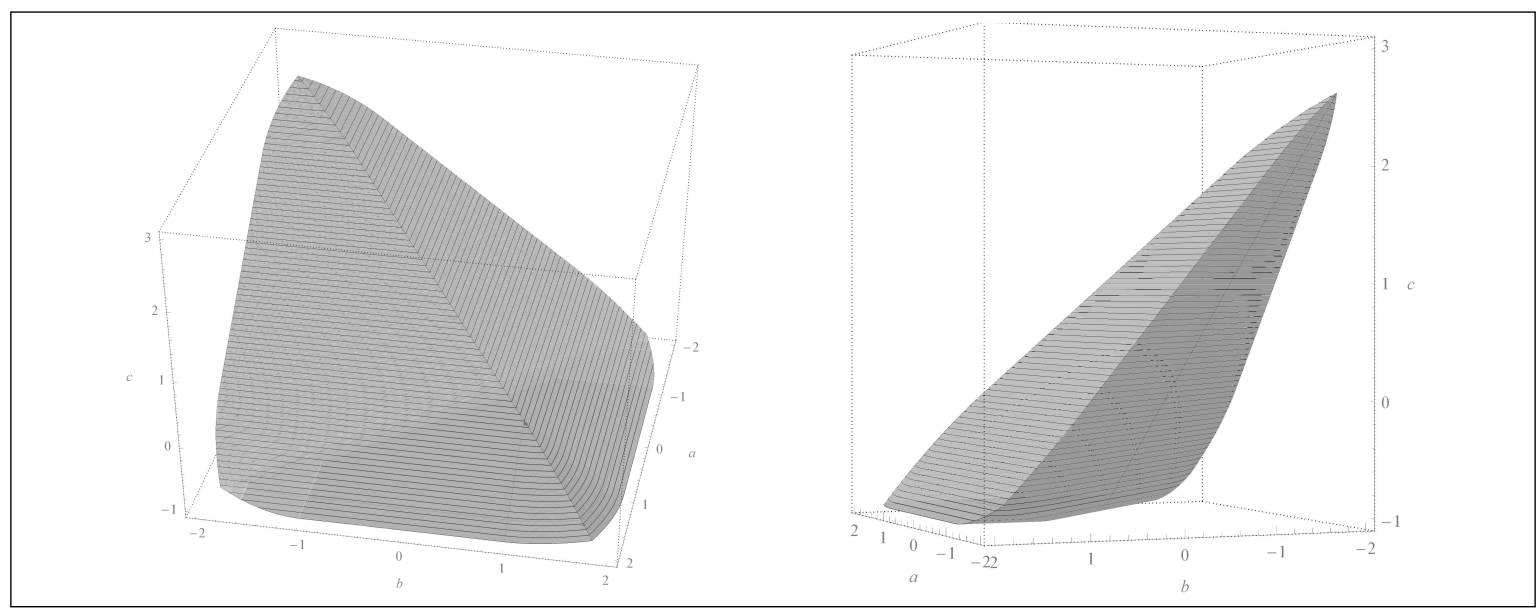

Figure 2: 3D plots of the parameter set PC5 from two different viewpoints.

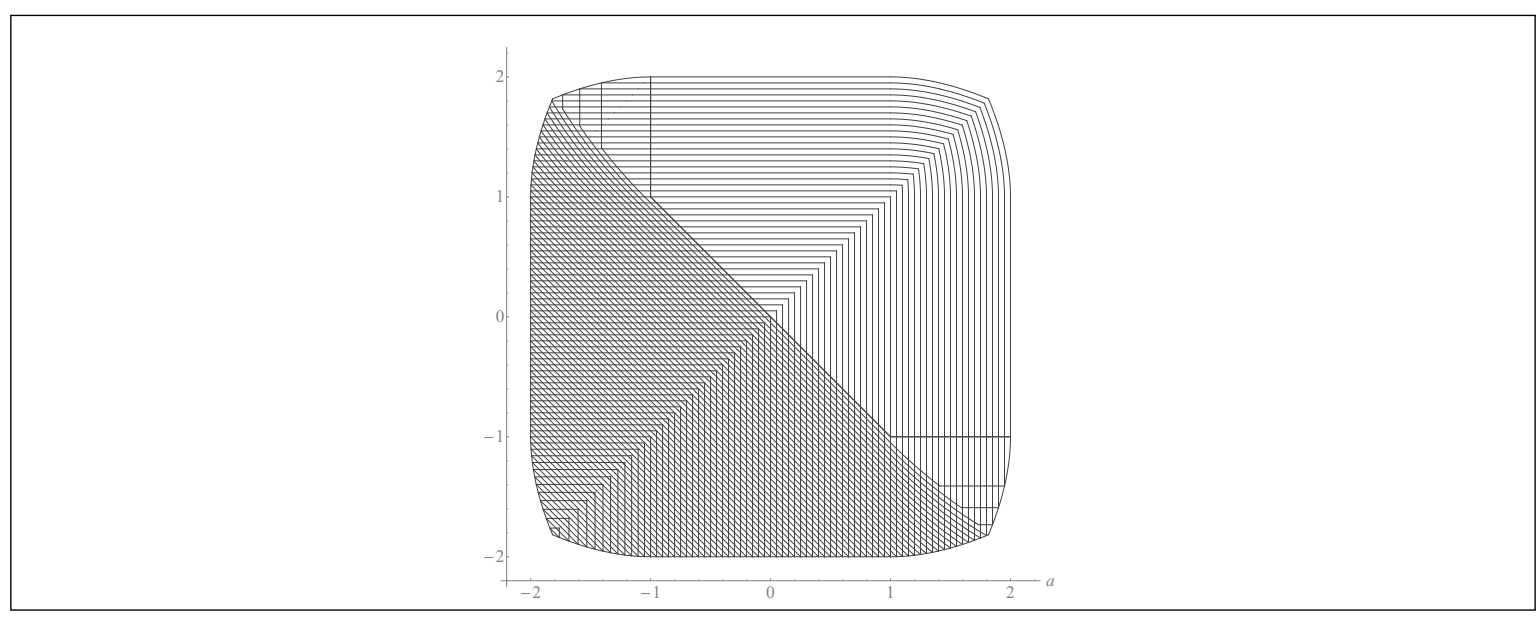

Figure 3: Contour plot of the parameter set PC5.

(ii) the corresponding range of $a$ (given $c$ ),

(iii) the corresponding lower and the upper bound for $b$ (given $c$ and $a$ ).

Table 1 gives a survey of the five cases which have to be distinguished for the values $c$ and, in Table 2, the complete formulas for the lower and upper bounds of $b$ (given $c$ and $a$ ) in each of these five cases are listed. To be precise, we summarize in each of the cases (I)-(V) the respective ranges of the level $c$ and of the value $a$ (given $c$ ). In (4.12)-(4.16) in Table 2 we give the complete formulas for the lower bounds $\mathrm{lb}_{(\mathrm{N})}^{[c]}(a)$ and the upper bounds $\mathrm{ub}_{(\mathrm{N})}^{[c]}(a)$ of the value $b$ (given $(c, a)$ ) such that $(a, b, c) \in \mathbf{P C} 5^{[c]}$.

When looking at the Cases (I)-(V) in Table 1 we immediately see that there is a minimal and a maximal value of $c$ such that there exists a parameter triplet $(a, b, c) \in \mathbf{P C 5}$ :

$$
\begin{aligned}
& \zeta_{\star}=\inf \{c \in \mathbb{R} \mid \text { there exist } a, b \in \mathbb{R} \text { such that }(a, b, c) \in \mathbf{P C 5}\}=-1, \\
& \zeta^{\star}=\sup \{c \in \mathbb{R} \mid \text { there exist } a, b \in \mathbb{R} \text { such that }(a, b, c) \in \mathbf{P C 5}\}=1+2 \sqrt{\frac{2}{3}} .
\end{aligned}
$$

The level set $\mathbf{P C} 5^{\left[\zeta^{*}\right]}=\mathbf{P C} 5^{[-1]}$ is visualized in Figure 4 (the darker set on the right-hand side), while the level set $\mathbf{P C} 5^{\left[\zeta^{\star}\right]}$ turns out to be a singleton:

$$
\mathbf{P C} 5^{\left[\zeta^{\star}\right]}=\mathbf{P C} 5^{[1+2 \sqrt{2 / 3}]}=\left\{\left(-1-\sqrt{\frac{2}{3}},-1-\sqrt{\frac{2}{3}}, 1+2 \sqrt{\frac{2}{3}}\right)\right\} .
$$



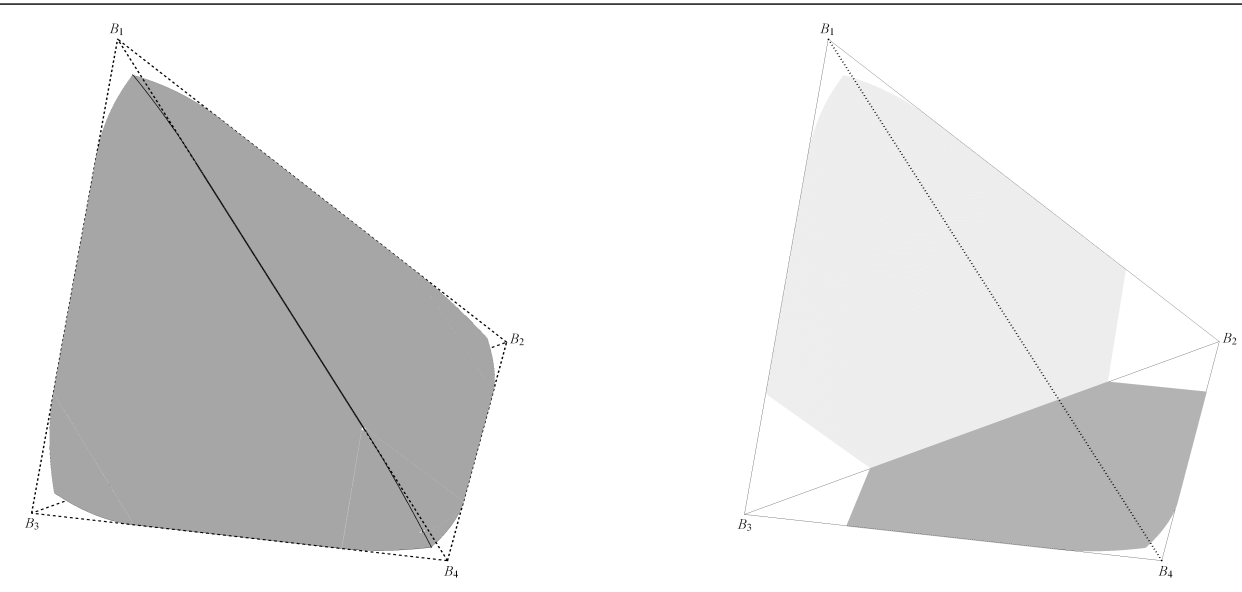

Figure 4: The parameter set PC5 (gray) and the polyhedron $\mathbf{P}_{\left(B_{1}, B_{2}, B_{3}, B_{4}\right)}$ (dashed edges, left), and the dark gray area in the base and the light gray area in the back of $\mathbf{P}_{\left(B_{1}, B_{2}, B_{3}, B_{4}\right)}$ indicating which parts of base and back of $\mathbf{P}_{\left(B_{1}, B_{2}, B_{3}, B_{4}\right)}$ coincide with the base and the back side of $\mathbf{P C 5}$, respectively (right).

A closer look at Cases (I)-(V), in particular at Case (IV), shows that the level set PC5 ${ }^{[\zeta]}$ and the corresponding level set of the polyhedron $\mathbf{P}_{\left(B_{1}, B_{2}, B_{3}, B_{4}\right)}$ given by (4.5) coincide if and only if $\zeta \in[0,2]$ :

Table 1: Level sets of the parameter set PC5

\begin{tabular}{|c|c|c|c}
\hline Case & Range of $c$ & Range of $a$ (given $c$ ) & Range of $b$ (given $(c, a)$ ) \\
\hline \hline (I) & {$\left[-1,-\frac{6}{7}[\right.$} & {$\left[\frac{1}{2}\left(-3-c-\sqrt{12-3(c-1)^{2}}\right), 1-c\right]$} & {$\left[\mathrm{lb}_{(\mathrm{I})}^{[c]}(a), \mathrm{ub}_{(\mathrm{I})}^{[c]}(a)\right]$} \\
\hline (II) & {$\left[-\frac{6}{7},-\sqrt{\frac{2}{3}}[\right.$} & {$\left[\frac{1}{2}\left(-3-c-\sqrt{12-3(c-1)^{2}}\right), 1-c\right]$} & {$\left[\mathrm{lb}_{(\mathrm{II})}^{[c]}(a), \mathrm{ub}_{(\mathrm{II})}^{[c]}(a)\right]$} \\
\hline (III) & {$\left[-\sqrt{\frac{2}{3}}, 0[\right.$} & {$\left[\frac{1}{2}\left(-1-c-\sqrt{12-3(c+1)^{2}}\right), 1-c\right]$} & {$\left[\mathrm{lb}_{(\mathrm{III})}^{[c]}(a), \mathrm{ub}\right.$} \\
\hline (III) & $[a)]$ \\
\hline (IV) & {$[0,2[-2,1-c]$} & {$\left[\mathrm{lb}_{(\mathrm{IV})}^{[c]}(a), \mathrm{ub}\right.$} \\
\hline (IV) & {$\left[2,1+2 \sqrt{\frac{2}{3}}\right]$} & $\left.\left[\frac{1}{2}\left(1-c-\sqrt{12-3(c-1)^{2}}\right)\right), \frac{1}{2}\left(-3-c+\sqrt{12-3(c-1)^{2}}\right)\right]$ & {$\left[\mathrm{lb}_{(\mathrm{V})}^{[c]}(a), \mathrm{ub}_{(\mathrm{V})}^{[c]}(a)\right]$} \\
\hline
\end{tabular}

Corollary 4.2. Consider for each $\zeta \in[-1,3]$ the level set

$$
\mathbf{P}_{\left(B_{1}, B_{2}, B_{3}, B_{4}\right)}^{[[]}=\operatorname{Conv}\left(\left\{\left(-2, \zeta-\frac{1}{2}, \zeta\right),\left(\zeta-\frac{1}{2}, \zeta-\frac{1}{2}, \zeta\right),\left(\zeta-\frac{1}{2}, 2, \zeta\right)\right\}\right)
$$

of the polyhedron $\mathbf{P}_{\left(B_{1}, B_{2}, B_{3}, B_{4}\right)}$ given in (4.5). Then we have:

$$
\mathbf{P C} 5^{[\zeta]}=\mathbf{P}_{\left(B_{1}, B_{2}, B_{3}, B_{4}\right)}^{[\zeta]} \quad \text { if and only if } \quad \zeta \in[0,2] .
$$


Table 2: Analytical description of the level sets $\mathbf{P C} 5^{[c]}$ of the parameter set PC5

Case (I): The functions $\mathrm{lb}_{(\mathrm{I})}^{[c]}, \mathrm{ub}_{(\mathrm{I})}^{[c]}:\left[\frac{1}{2}\left(-3-c-\sqrt{12-3(c-1)^{2}}\right), 1-c\right] \rightarrow \mathbb{R}$ specify, for each $c \in\left[-1,-\frac{6}{7}[\right.$, the lower and upper bounds of the parameter $b$ (in dependence on $c$ and $a$ ):

$$
\begin{aligned}
& \operatorname{lb}_{(\mathrm{I})}^{[c]}(a)= \begin{cases}\frac{1}{2}\left(3-a-c-\sqrt{12-3(a+c+1)^{2}}\right) & \text { if } a \in\left[\frac{1}{2}\left(-3-c-\sqrt{12-3(c-1)^{2}}\right),-2-c[,\right. \\
-1-a-c & \text { if } a \in[-2-c, 1[, \\
\frac{1}{2}\left(-a-2 c-\sqrt{12-3(a-2)^{2}}\right) & \text { if } a \in\left[1, \frac{1}{2}\left(3+c+\sqrt{12-3(c-1)^{2}}\right)[,\right. \\
\frac{1}{2}\left(-3-c-\sqrt{12-3(c-1)^{2}}\right) & \text { otherwise, }\end{cases} \\
& \mathrm{ub}_{(\mathrm{I})}^{[c]}(a)= \begin{cases}1-c & \text { if } a \in\left[\frac{1}{2}\left(-3-c-\sqrt{12-3(c-1)^{2}}\right), 1[,\right. \\
\frac{1}{2}\left(-a-2 c+\sqrt{12-3(a-2)^{2}}\right) & \text { if } a \in\left[1, \frac{1}{6}\left(3-3 c+\sqrt{36-3(c+3)^{2}}\right)[,\right. \\
\frac{1}{2}\left(3-a-c+\sqrt{12-3(a+c+1)^{2}}\right) & \text { otherwise. }\end{cases}
\end{aligned}
$$

Case (II): The functions $\mathrm{lb}_{(\mathrm{II})}^{[c]}, \mathrm{ub}_{(\mathrm{II})}^{[c]}:\left[\frac{1}{2}\left(-3-c-\sqrt{12-3(c-1)^{2}}\right), 1-c\right] \rightarrow \mathbb{R}$ specify, for each $c \in\left[-\frac{6}{7},-\sqrt{\frac{2}{3}}[\right.$, the lower and upper bounds of the parameter $b$ (in dependence on $c$ and $a$ ):

$$
\begin{aligned}
& \mathrm{lb}_{(\mathrm{II})}^{[c]}(a)= \begin{cases}\frac{1}{2}\left(3-a-c-\sqrt{12-3(a+c+1)^{2}}\right) & \text { if } a \in\left[\frac{1}{2}\left(-3-c-\sqrt{12-3(c-1)^{2}}\right),-2-c[,\right. \\
-1-a-c & \text { if } a \in[-2-c, 1[, \\
\frac{1}{2}\left(-a-2 c-\sqrt{12-3(a-2)^{2}}\right) & \text { if } a \in\left[1, \frac{1}{2}\left(3+c+\sqrt{12-3(c-1)^{2}}\right)[,\right. \\
\frac{1}{2}\left(-3-c-\sqrt{12-3(c-1)^{2}}\right) & \text { otherwise, }\end{cases} \\
& \mathrm{ub}_{(\mathrm{III})}^{[c]}(a)= \begin{cases}1-c & \text { if } a \in\left[\frac{1}{2}\left(-3-c-\sqrt{12-3(c-1)^{2}}\right), 1[,\right. \\
\frac{1}{2}\left(-a-2 c+\sqrt{12-3(a-2)^{2}}\right) & \text { if } a \in\left[1, \frac{1}{6}\left(3-3 c+\sqrt{36-3(c+3)^{2}}\right)[,\right. \\
\frac{1}{2}\left(3-a-c+\sqrt{12-3(a+c+1)^{2}}\right) & \text { otherwise. }\end{cases}
\end{aligned}
$$

Case (III): The functions $\mathrm{lb}_{(\mathrm{III})}^{[c]}, \mathrm{ub}_{(\mathrm{III})}^{[c]}:\left[\frac{1}{2}\left(-1-c-\sqrt{12-3(c+1)^{2}}\right), 1-c\right] \rightarrow \mathbb{R}$ specify, for each $c \in\left[-\sqrt{\frac{2}{3}}, 0[\right.$, the lower and upper bounds of the parameter $b$ (in dependence on $c$ and $a$ ):

$$
\begin{aligned}
& \mathrm{lb}_{(\mathrm{III})}^{[c]}(a)= \begin{cases}\frac{1}{2}\left(3-a-c-\sqrt{12-3(a+c+1)^{2}}\right) & \text { if } a \in\left[\frac{1}{2}\left(-1-c-\sqrt{12-3(c+1)^{2}}\right),-2-c[,\right. \\
-1-a-c & \text { if } a \in[-2-c, 1[, \\
\frac{1}{2}\left(-a-2 c-\sqrt{12-3(a-2)^{2}}\right) & \text { otherwise, }\end{cases} \\
& \mathrm{ub}_{(\mathrm{III})}^{[c]}(a)= \begin{cases}1-c & \text { if } a \in\left[\frac{1}{2}\left(-1-c-\sqrt{12-3(c+1)^{2}}\right), 1[,\right. \\
\frac{1}{2}\left(-a-2 c+\sqrt{12-3(a-2)^{2}}\right) & \text { if } a \in\left[1, \frac{1}{6}\left(3-3 c+\sqrt{36-3(c+3)^{2}}\right)[,\right. \\
\frac{1}{2}\left(3-a-c+\sqrt{12-3(a+c+1)^{2}}\right) & \text { otherwise. }\end{cases}
\end{aligned}
$$

Case (IV): The functions $\mathrm{lb}_{(\mathrm{IV})}^{[c]}, \mathrm{ub}_{(\mathrm{IV})}^{[c]}:[-2,1-c] \rightarrow \mathbb{R}$ specify, for each $c \in[0,2[$, the lower and upper bounds of the parameter $b$ (in dependence on $c$ and $a$ ):

$$
\operatorname{lb}_{(\mathrm{IV})}^{[c]}(a)=-1-a-c, \quad \quad \mathrm{ub}_{(\mathrm{IV})}^{[c]}(a)=1-c .
$$

Case (V): The functions $\mathrm{lb}_{(\mathrm{V})}^{[c]}, \mathrm{ub}_{(\mathrm{V})}^{[c]}:\left[\frac{1}{2}\left(1-c-\sqrt{12-3(c-1)^{2}}\right), \frac{1}{2}\left(-3-c+\sqrt{12-3(c-1)^{2}}\right)\right] \rightarrow \mathbb{R}$ specify, for each $c \in\left[2,1+2 \sqrt{\frac{2}{3}}\right]$, the lower and upper bounds of the parameter $b$ (in dependence on $c$ and $a$ ):

$$
\mathrm{lb}_{(\mathrm{V})}^{[c]}(a)=-1-a-c, \quad \quad \mathrm{ub}_{(\mathrm{V})}^{[c]}(a)=\frac{1}{2}\left(-3-c+\sqrt{12-3(c-1)^{2}}\right)
$$




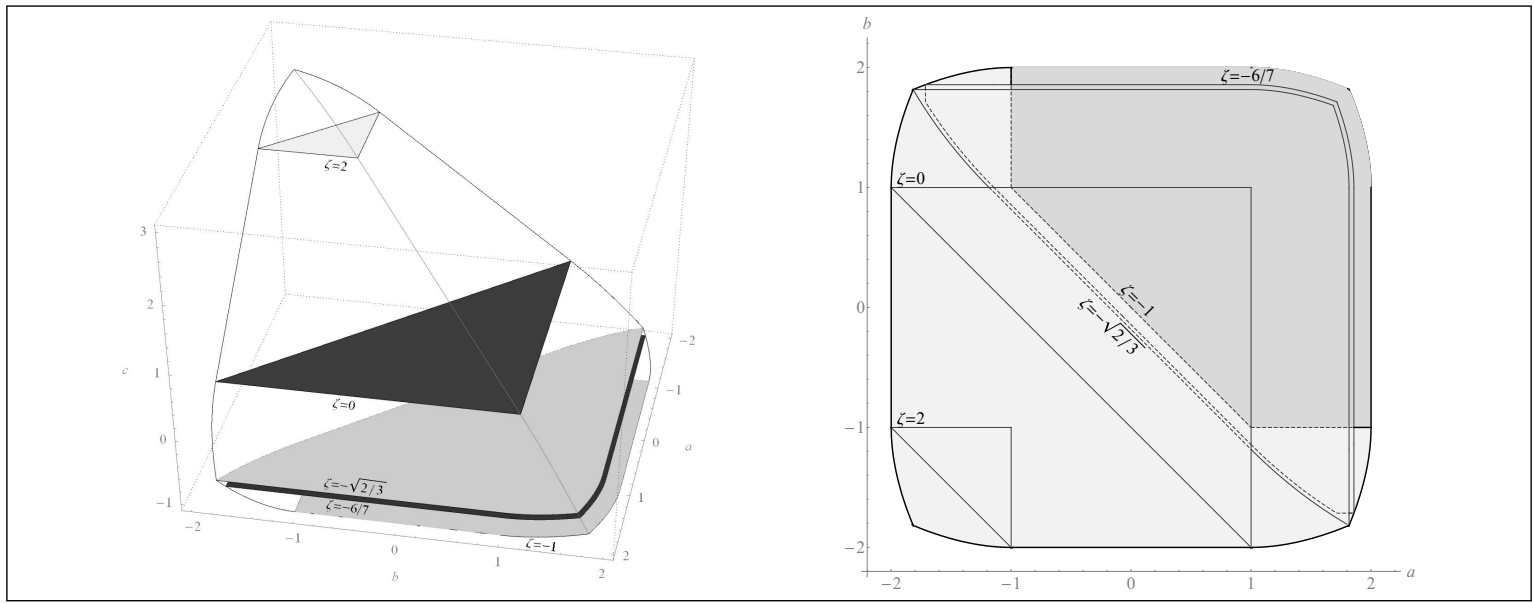

Figure 5: 3D plot of several level sets of PC5: $\zeta=-1$ (light gray), $\zeta=-6 / 7$ (dark gray), $\zeta=-\sqrt{2 / 3}$ (light gray), $\zeta=0$ (dark gray), and $\zeta=2$ (light gray, left), and the projection of PC5 to the $a b$-plane (light gray) including the bottom of PC5 (i.e., the level set at $\zeta=0$ ) marked in gray, and the contours of the level sets at $\zeta \in\{-6 / 7,-\sqrt{2 / 3}, 0,2\}$, representing the bounds of the cases (I)-(V) in Tables 1-2 (right).

Figure 4 (right) illustrates which parts of the back side and the base of the polyhedron $\mathbf{P}_{\left(B_{1}, B_{2}, B_{3}, B_{4}\right)}$ (i.e., the triangles determined by the points $B_{1}-B_{3}$ and $B_{2}-B_{4}$, respectively) coincide with the back side and the bottom of the parameter set PC5.

Using again the quantifier elimination of Mathematica we see that the set $\mathbf{P C}_{\text {sym }} \subseteq$ PC5 given by (4.1), i.e., the intersection of the parameter set PC5 and the plane characterized by $a-b=0$ (see Figure 7), can be characterized by

$$
\mathbf{P C 5}_{\text {sym }}=\left\{(a, a, c) \in \mathbf{P C 5} \mid \operatorname{glbdiag}(a) \leq c \leq \operatorname{lubdiag}(a) \text { for each } a \in\left[-1-\sqrt{\frac{2}{3}}, 1+\sqrt{\frac{2}{3}}\right]\right\},
$$

where the functions glbdiag, lubdiag: $\left[-1-\sqrt{\frac{2}{3}}, 1+\sqrt{\frac{2}{3}}\right] \rightarrow \mathbb{R}$ are defined by, respectively,

$$
\begin{aligned}
& \operatorname{glbdiag}(a)=\max (-2 a-1,-1), \\
& \operatorname{lubdiag}(a)= \begin{cases}\frac{1}{2}\left(-a+\sqrt{-12 a-3 a^{2}}\right) & \text { if } a \in\left[-1-\sqrt{\frac{2}{3}},-1[,\right. \\
1-a & \text { if } a \in[-1,1[, \\
\frac{1}{2}\left(-3 a+\sqrt{12 a-3 a^{2}}\right) & \text { if } a \in\left[1,1+\sqrt{\frac{2}{3}}\right] .\end{cases}
\end{aligned}
$$

Numerical integration tells us that the volume of the parameter set PC5 has the approximate value of 10.5184 cubic units. Since the volume of the pyramid $\mathbf{P}_{\left(B_{1}, B_{2}, B_{3}, B_{4}\right)}$ (4.5) equals $\frac{32}{3}$ cubic units, the volume of PC5 corresponds to approximately $98.6102 \%$ of the volume of $\mathbf{P}_{\left(B_{1}, B_{2}, B_{3}, B_{4}\right)}$. The distribution of the areas of the level sets PC5 ${ }^{[\zeta]}$ of PC5 on the interval $[-1,1+2 \sqrt{2 / 3}]$ (in comparison to the areas of the level sets of the polyhedron $\left.\mathbf{P}_{\left(B_{1}, B_{2}, B_{3}, B_{4}\right)}\right)$ is visualized in Figure 6 .

Apparently, the projection of the parameter set PC5 to the $a b$-plane is not only symmetric with respect to the main diagonal $a-b=0$ (which follows from (2.13) by construction). It seems (see Figure 5) that this projection of PC5 is also symmetric with respect to the opposite diagonal $a+b=0$ and to the lines determined by $a=0$ and $b=0$. 


\section{Polynomial copulas of degree five satisfying some particular (in)equalities}

\subsection{Symmetric polynomial copulas of degree five}

A copula $C:[0,1]^{2} \rightarrow[0,1]$ is called symmetric if $C(x, y)=C(y, x)$ for each $(x, y) \in[0,1]^{2}$. Symmetric copulas are closely related to exchangeable random variables $X$ and $Y$, i.e., where the random vectors $(X, Y)$ and $(Y, X)$ are identically distributed.

To be precise (see [66, Theorem 2.7.4]), if $(\Omega, \mathcal{A}, P)$ is a probability space then two continuous random variables $X, Y: \Omega \rightarrow \mathbb{R}$ are exchangeable if and only if they have the same marginal distributions and if the corresponding copula $C:[0,1]^{2} \rightarrow[0,1]$ according to Theorem 2.1 is symmetric.

Taking into account the result of [92] and the structure of the formula for $C_{(a, b, c)}$ in (2.13), we obtain the following obvious result:

Corollary 5.1. Let $C_{(a, b, c)}:[0,1]^{2} \rightarrow[0,1]$ be a polynomial copula of degree 5 , i.e., $(a, b, c) \in \mathbf{P C 5}$. The copula $C_{(a, b, c)}$ is symmetric if and only if $a=b$, i.e., if $(a, b, c) \in \mathbf{P C} 5_{\mathrm{sym}}$.

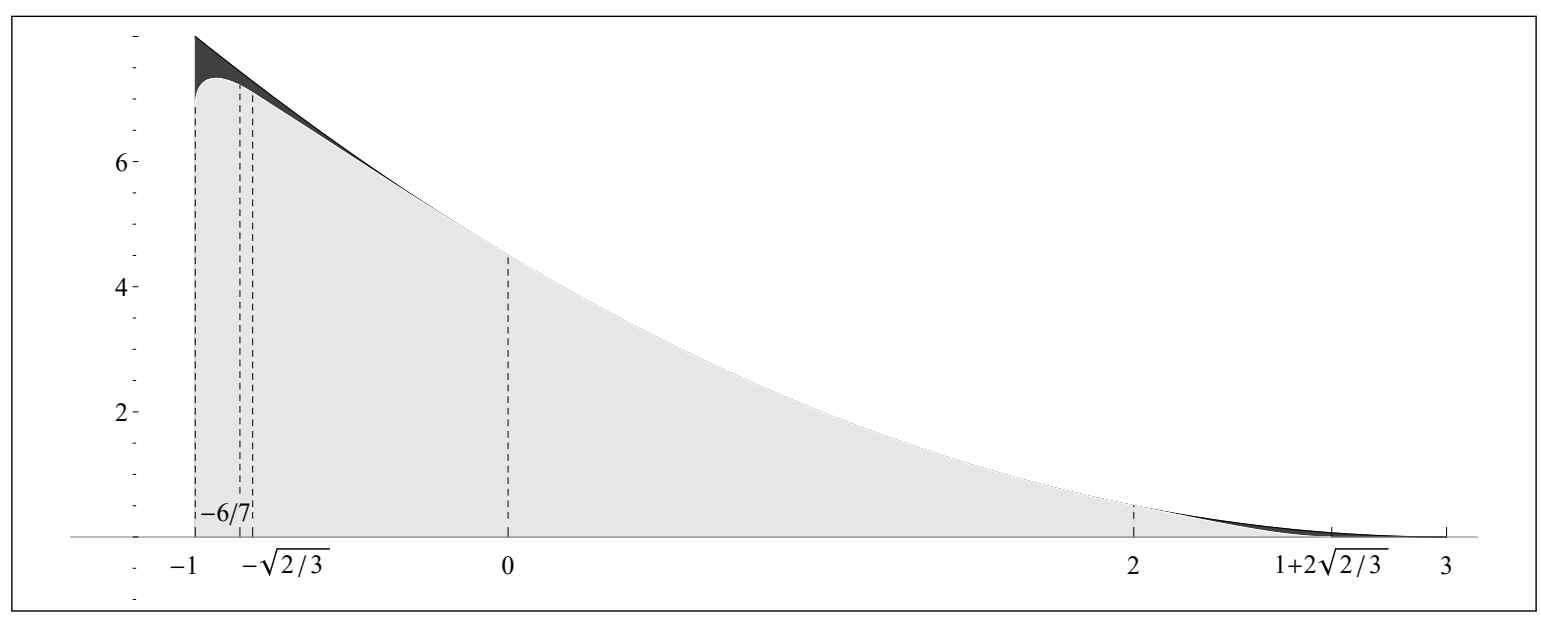

Figure 6: The areas of the level sets of the parameter set PC5 on the interval $[-1,1+2 \sqrt{2 / 3}]$ (light gray) compared with the areas of the level sets (dark gray) of the polyhedron $\mathbf{P}_{\left(B_{1}, B_{2}, B_{3}, B_{4}\right)}$.

The set $\mathbf{P C} 5_{\text {sym }}$ as given in (4.17) is visualized in Figure 7. Note that each $(a, a, c) \in \mathbf{P C} 5_{\text {sym }}$ can also be characterized as follows:

$$
a \in \begin{cases}{\left[\frac{-1-c}{2}, \frac{3-3 c+\sqrt{36-3(c+3)^{2}}}{6}\right]} & \text { if } c \in[-1,0[, \\ {\left[\frac{-1-c}{2}, 1-c\right]} & \text { if } c \in[0,2[, \\ {\left[\frac{-1-c}{2}, \frac{-3-c+\sqrt{12-3(1-c)^{2}}}{2}\right]} & \text { if } c \in\left[2,1+2 \sqrt{\frac{2}{3}}\right] .\end{cases}
$$




\subsection{Schur concave polynomial copulas of degree five}

Schur convexity and Schur concavity (as its dual) were introduced in [80] as variants of the convexity of real functions (see also [74]). Schur convex functions preserve a particular preorder called majorization [61] and play a role in some related inequalities [87].

A function $f:[0,1]^{2} \rightarrow[0,1]$ is called Schur concave if for all $(x, y),(u, v) \in[0,1]^{2}$ we have

$$
x+y=u+v \quad \text { and } \quad \min (x, y) \leq \min (u, v) \quad \Longrightarrow \quad f(x, y) \leq f(u, v) .
$$

In particular, a copula $C:[0,1]^{2} \rightarrow[0,1]$ is Schur concave (see $\left.[47-49,77]\right)$ if and only if, for all $(x, y) \in[0,1]^{2}$ and all $\lambda \in[0,1]$,

$$
C(x, y) \leq C(\lambda x+(1-\lambda) y,(1-\lambda) x+\lambda y) .
$$

Clearly, the three basic copulas $W, \Pi$ and $M$ are Schur concave, as well as each associative copula (see [29, 47]).

It turns out that the set of Schur concave polynomial copulas of degree 5 coincides with the set of symmetric polynomial copulas of degree 5 :

Proposition 5.2. Let $C_{(a, b, c)}:[0,1]^{2} \rightarrow[0,1]$ be a polynomial copula of degree 5 , i.e., $(a, b, c) \in \mathbf{P C} 5$. The following are equivalent:

(i) $C_{(a, b, c)}$ is Schur concave;

(ii) $C_{(a, b, c)}$ is symmetric, i.e., $a=b$.

Proof. Since each Schur concave copula is symmetric [29], each Schur concave polynomial copula $C_{(a, b, c)}$ satisfies $(a, b, c) \in \mathbf{P C} 5_{\text {sym }}$, i.e., $a=b$, which means that (i) implies (ii).

In order to show that also (ii) implies (i) we have to consider points $(x, y) \in[0,1]^{2}$ with a fixed sum $u=x+y$, i.e., $u \in[0,2]$. Observe that $u=0$ and $u=2$ are equivalent to $(x, y)=(0,0)$ and $(x, y)=(1,1)$, respectively, so we may restrict ourselves to $u \in$ ]0, 2[ because the Schur concavity on a single point is trivial. Since in this part of the proof our copulas are assumed to be symmetric we may also restrict ourselves to the upper left triangle $\Delta=\left\{(x, y) \in[0,1]^{2} \mid x \leq y\right\}$ of the unit square $[0,1]^{2}$. Then, for a fixed $\left.u \in\right] 0,2[$ we have

$$
((x, y) \in \Delta \quad \text { and } \quad x+y=u) \Longleftrightarrow\left(x \in\left[\max (u-1,0), \frac{u}{2}\right] \text { and } y=u-x\right) .
$$

Then a symmetric copula $C:[0,1]^{2} \rightarrow[0,1]$ is Schur concave if and only if for each $\left.u \in\right] 0,2[$ the onedimensional section $C(\cdot, u-\cdot):\left[\max (u-1,0), \frac{u}{2}\right] \rightarrow \mathbb{R}$ given by $(C(\cdot, u-\cdot))(x)=C(x, u-x)$ is strictly increasing. For a symmetric polynomial copula $C_{(a, a, c)}$ of degree 5, i.e., with $(a, a, c) \in \mathbf{P C} 5_{\text {sym }}$, and for each $u \in$ ]0,2[ this means that the derivative of this section of $C_{(a, a, c)}$ with respect to $x$ is non-negative or, equivalently and in a formal way, for each $(u, x) \in] 0,2\left[\times\left[\max (u-1,0), \frac{u}{2}\right]\right.$ we have

$$
\frac{\partial C_{(a, a, c)}(x, u-x)}{\partial x}=u-2 x+(a u+c)\left(4 x^{3}-6 u x^{2}+2 x\left(u^{2}+u-1\right)+u-u^{2}\right) \geq 0 .
$$

Rewriting this in Mathematica code,

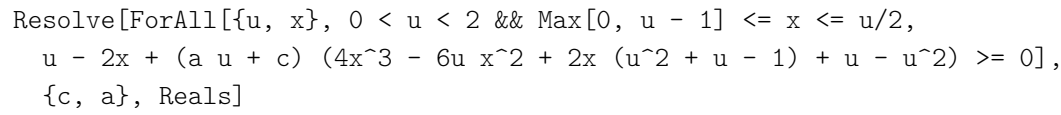

we obtain that for each pair $(a, c) \in \mathbb{R}^{2}$ satisfying $c \geq \max (-1-2 a,-1)$ the inequality (5.2) holds for all $(u, x) \in] 0,2\left[\times\left[\max (u-1,0), \frac{u}{2}\right]\right.$. As a consequence of

$$
\mathbf{P C 5}_{\text {sym }} \subseteq\left\{(a, a, c) \in \mathbb{R}^{3} \mid c \geq \max (-1-2 a,-1)\right\},
$$

this means that also (ii) implies (i). 


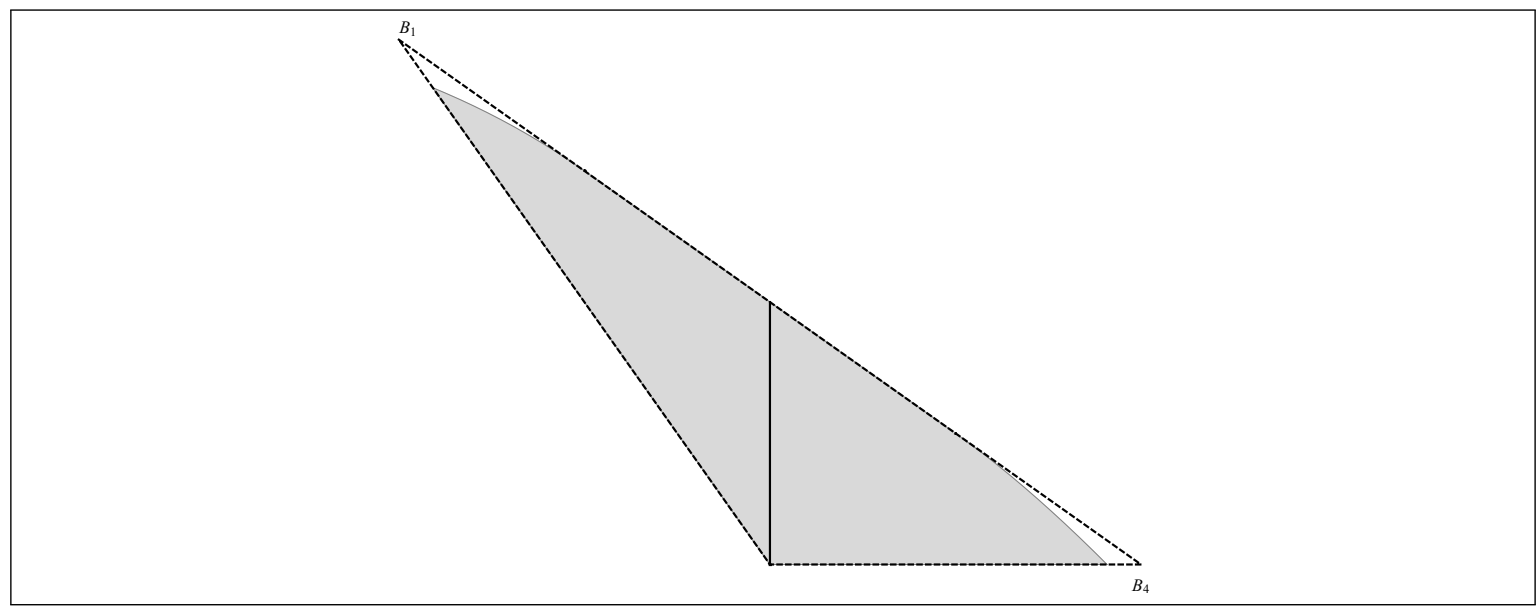

Figure 7: The intersection of the plane $a-b=0$ with $\mathbf{P}_{\left(B_{1}, B_{2}, B_{3}, B_{4}\right)}$ (dashed triangle) and with PC5 (gray area) showing the parameters of symmetric (and Schur concave) polynomial copulas of degree 5 together with the thick line segment marking the position of the Eyraud-Farlie-Gumbel-Morgenstern copulas.

\subsection{Ultramodular polynomial copulas of degree five}

If $A$ is a non-empty subset of $\mathbb{R}^{n}$ then a function $f: A \rightarrow \mathbb{R}^{n}$ is called ultramodular [60] if its increments are non-decreasing. This means that for all $\mathbf{u}, \mathbf{v} \in A$ with $\mathbf{u} \leq \mathbf{v}$ and for all $\mathbf{h} \in \mathbb{R}^{n}$ such that $\mathbf{u}+\mathbf{h} \in A$ and $\mathbf{v}+\mathbf{h} \in A$ we have

$$
f(\mathbf{u}+\mathbf{h})-f(\mathbf{u}) \leq f(\mathbf{v}+\mathbf{h})-f(\mathbf{v}) .
$$

In the special case $n=2$, a copula $C:[0,1]^{2} \rightarrow[0,1]$ is ultramodular (see $[47-49,77]$ ) if and only if for all $\left(u_{1}, u_{2}\right),\left(v_{1}, v_{2}\right),\left(w_{1}, w_{2}\right) \in[0,1]^{2}$ satisfying $\left(u_{1}, u_{2}\right)+\left(v_{1}, v_{2}\right)+\left(w_{1}, w_{2}\right) \in[0,1]^{2}$ the following inequality holds:

$$
C\left(u_{1}+w_{1}, u_{2}+w_{2}\right)-C\left(u_{1}, u_{2}\right) \leq C\left(u_{1}+v_{1}+w_{1}, u_{2}+v_{2}+w_{2}\right)-C\left(u_{1}+v_{1}, u_{2}+v_{2}\right) .
$$

In a geometrical formulation, an ultramodular copula $C$ is characterized by the fact that all onedimensional vertical and horizontal sections are convex (see [60, Corollary 4.1] and [48, Proposition 2.3]) or, equivalently, that for all $(x, y) \in[0,1]^{2}$ where the second partial derivatives exist we have

$$
\frac{\partial^{2} C(x, y)}{\partial x^{2}} \geq 0 \text { and } \frac{\partial^{2} C(x, y)}{\partial y^{2}} \geq 0
$$

The concepts of ultramodular copulas and of stochastically decreasing copulas $[5,57,66,86]$ are equivalent (see Subsection 5.6 for some details).

Proposition 5.3. Let $C_{(a, b, c)}:[0,1]^{2} \rightarrow[0,1]$ be a polynomial copula of degree 5, i.e., $(a, b, c) \in$ PC5. The following are equivalent:

(i) The copula $C_{(a, b, c)}$ is ultramodular.

(ii) The triplet $(a, b, c)$ is an element of the subset $\mathbf{P}_{\mathrm{UM}}$ of $\mathbf{P C} 5$ given by

$$
\begin{aligned}
\mathbf{P}_{\mathrm{UM}}=\operatorname{Conv}\left(\left\{(0,0,0),\left(-\frac{1}{3},-\frac{1}{3},-\frac{1}{3}\right),\left(-\frac{1}{2}, 0,-\frac{1}{2}\right),\left(0,-\frac{1}{2},-\frac{1}{2}\right),\right.\right. \\
\left.\left.\left(\frac{1}{2},-\frac{1}{2},-1\right),\left(-\frac{1}{2}, \frac{1}{2},-1\right),\left(\frac{1}{2}, 0,-1\right),\left(0, \frac{1}{2},-1\right),\left(\frac{1}{3}, \frac{1}{3},-1\right)\right\}\right) .
\end{aligned}
$$

Proof. If $C_{(a, b, c)}$ as given by (2.13) is an ultramodular polynomial copula of degree 5 then $C_{(a, b, c)}$ must satisfy (5.3) for each $(x, y) \in[0,1]^{2}$. Compute the second partial derivatives of $C_{(a, b, c)}$

$$
\frac{\partial^{2} C_{(a, b, c)}(x, y)}{\partial x^{2}}=2 y(y-1)(3 a x+b y-a+c), \quad \frac{\partial^{2} C_{(a, b, c)}(x, y)}{\partial y^{2}}=2 x(x-1)(a x+3 b y-b+c),
$$




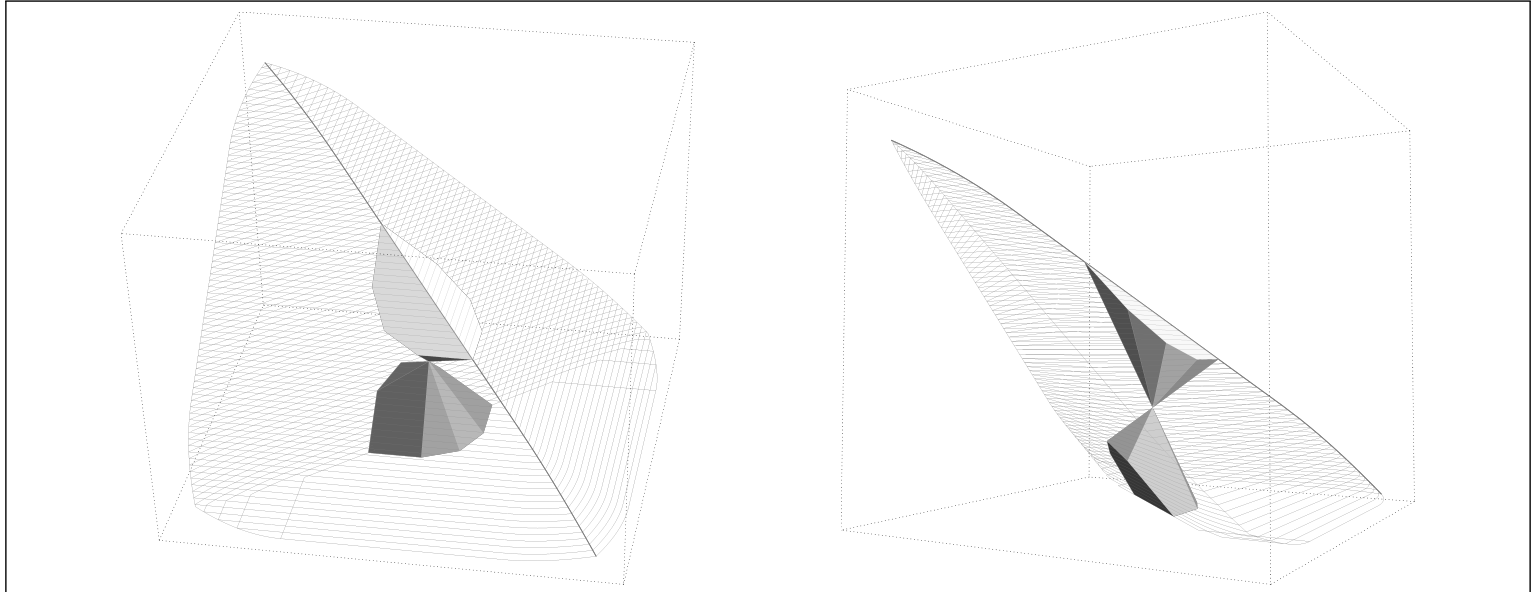

Figure 8: Parameter sets $\mathbf{P}_{\mathrm{UM}}=\mathbf{P}_{\mathrm{SD}}$ of ultramodular (or, equivalently, stochastically decreasing) polynomial copulas of degree 5 (bottom) and $\mathbf{P}_{\mathrm{SI}}$ (see Corollary 5.9) of stochastically increasing polynomial copulas of degree 5 (top) from two different viewpoints (the set PC5 is indicated by the gray skeleton).

and take into account the continuity of the polynomials occurring in these second partial derivatives and the fact that $x(1-x)$ and $y(1-y)$ are strictly negative on $] 0,1[$ and vanish for $x \in\{0,1\}$ and $y \in\{0,1\}$, respectively. Then we see that the validity of (5.3) means that for each $(x, y) \in[0,1]^{2}$ we have

$$
3 a x+b y-a+c \leq 0 \quad \text { and } \quad a x+3 b y-b+c \leq 0 .
$$

If we insert into (5.5) the four points in $(x, y) \in\{0,1\}^{2}$ we obtain the following system of linear inequalities which has to be satisfied by any triplet $(a, b, c) \in \mathbf{P C} 5$ corresponding to an ultramodular polynomial copula of degree 5:

$$
\begin{array}{llll}
-a+c \leq 0, & -a+b+c \leq 0, & 2 a+c \leq 0, & 2 a+b+c \leq 0, \\
-b+c \leq 0, & a-b+c \leq 0, & 2 b+c \leq 0, & a+2 b+c \leq 0 .
\end{array}
$$

Since $(a, b, c) \in \mathbf{P}_{\left(B_{1}, B_{2}, B_{3}, B_{4}\right)}$ because of (4.7), ( $\left.a, b, c\right)$ must also solve the system of linear inequalities (4.6). Using the Mathematica command Reduce, we see that the set of solutions of the joint systems (5.6), (5.7) and (4.6) of linear inequalities coincides with the polyhedron $\mathbf{P}_{\mathrm{UM}}$ (5.4), i.e., $(a, b, c) \in \mathbf{P}_{\mathrm{UM}}$.

Since for each vertex $\left(a^{\star}, b^{\star}, c^{\star}\right)$ of $\mathbf{P}_{\mathrm{UM}}$ the density $\varphi_{C_{\left(a^{*}, b^{*}, c^{*}\right)}}$ is easily seen to be non-negative (i.e., satisfies (3.2) for each $\left.(x, y) \in[0,1]^{2}\right)$, we have $\left(a^{\star}, b^{\star}, c^{\star}\right) \in \mathbf{P C 5}$ and, because of the convexity of PC5, also $\mathbf{P}_{\mathrm{UM}} \subseteq$ PC5.

Conversely, if for the polynomial copula $C_{(a, b, c)}$ we have $(a, b, c) \in \mathbf{P}_{\mathrm{UM}}$, then $(a, b, c)$ solves the joint systems (5.6) and (5.7) of linear inequalities and, as a consequence, all horizontal and vertical one-dimensional sections of $C_{(a, b, c)}$ are convex, i.e., $C_{(a, b, c)}$ is ultramodular.

Obviously, the vertex $(0,0,0)$ of the convex set $\mathbf{P}_{\mathrm{UM}}$ corresponds to the product copula $\Pi$ which is the greatest ultramodular copula.

\subsection{Positive quadrant dependence}

If $(\Omega, \mathcal{A}, P)$ is a probability space, $X, Y: \Omega \rightarrow \mathbb{R}$ are continuous random variables and $C:[0,1]^{2} \rightarrow[0,1]$ is the corresponding copula according to Theorem 2.1, then $X$ and $Y$ (and $C$ ) are said to be positively quadrant dependent (PQD) $[57,66]$ if, for all $(u, v) \in \mathbb{R}^{2}$

$$
P[X \leq u, Y \leq v] \geq P[X \leq u] \cdot P[Y \leq v]
$$

or (recalling the product copula $\left.\Pi=C_{(0,0,0)}\right)$ if $C \geq \Pi$. 
Proposition 5.4. Let $C_{(a, b, c)}:[0,1]^{2} \rightarrow[0,1]$ be a polynomial copula of degree 5 , i.e., $(a, b, c) \in$ PC5. The following are equivalent:

(i) The copula $C_{(a, b, c)}$ is positively quadrant dependent.

(ii) The triplet $(a, b, c)$ is an element of the subset $\mathbf{P}_{\mathrm{PQD}}$ of $\mathbf{P C 5}$ given by

$$
\mathbf{P}_{\mathrm{PQD}}=\operatorname{Conv}(\{(-1,-1,2),(-1,0,1),(0,-1,1),(0,0,0),(0,1,0),(1,1,0),(1,0,0)\}) .
$$

Proof. Let us first determine which polynomial functions $C_{(a, b, c)}$ as given in (2.13) satisfy the two conditions $C_{(a, b, c)} \geq \Pi$ and $(a, b, c) \in \mathbf{P}_{\left(B_{1}, B_{2}, B_{3}, B_{4}\right)}$.

To verify $C_{(a, b, c)} \geq \Pi$ it suffices, because of the convexity of $[0,1]^{2}$ and the convexity of the function $(x, y) \longmapsto a x+b y+c$, to check the non-negativity of $a x+b y+c$ for each vertex $(x, y) \in\{0,1\}^{2}$ of the unit square, which exactly means that the triplet $(a, b, c)$ satisfies the following system of linear inequalities:

$$
a+c \geq 0, \quad b+c \geq 0, \quad a+b+c \geq 0, \quad c \geq 0 .
$$

We already know that $(a, b, c) \in \mathbf{P}_{\left(B_{1}, B_{2}, B_{3}, B_{4}\right)}$ if and only if $(a, b, c)$ solves the system (4.6) of linear inequalities.

Subtracting the fourth inequality from the sum of the first two inequalities in (4.6), the joint system of the inequalities (5.10) and (4.6) can be rewritten as

$$
0 \leq a+c \leq 1, \quad 0 \leq b+c \leq 1, \quad 0 \leq a+b+c \leq 2, \quad 0 \leq c \leq 3 .
$$

It is readily seen (e.g., using again a Mathematica command like Reduce) that $(a, b, c)$ solves (5.11) if and only if $(a, b, c) \in \mathbf{P}_{\mathrm{PQD}}$ as given by (5.9).

This means that $(a, b, c) \in \mathbf{P}_{\mathrm{PQD}}$ if and only if a polynomial function $C_{(a, b, c)}$ satisfies $C_{(a, b, c)} \geq \Pi$ and $(a, b, c) \in \mathbf{P}_{\left(B_{1}, B_{2}, B_{3}, B_{4}\right)}$.

Since the third component of each vertex of the polyhedron $\mathbf{P}_{\mathrm{PQD}}$ lies in the interval [0, 2] (where the sets PC5 and $\mathbf{P}_{\left(B_{1}, B_{2}, B_{3}, B_{4}\right)}$ coincide) we also have $\mathbf{P}_{\mathrm{PQD}} \subseteq \mathbf{P C 5}$, i.e., the polynomial function $C_{(a, b, c)}$ is indeed a positively quadrant dependent copula.

Then for $c \in \mathbb{R}$ the level set $\mathbf{P}_{\mathrm{PQD}}{ }^{[c]}$ of the parameter set $\mathbf{P}_{\mathrm{PQD}}$ of positively quadrant dependent polynomial copulas of degree 5 is given by

$$
\mathbf{P}_{\mathrm{PQD}}{ }^{[c]}= \begin{cases}\operatorname{Conv}(\{(0,-c, c),(-c, 0, c),(1-c,-c, c),(1-c, 1-c, c),(-c, 1-c, c)\}) & \text { if } c \in[0,1[, \\ \operatorname{Conv}(\{(1-c,-1, c),(1-c, 1-c, c),(-1,1-c, c)\}) & \text { if } c \in[1,2], \\ \emptyset & \text { otherwise. }\end{cases}
$$

\subsection{Negative quadrant dependence}

Negative quadrant dependence of continuous random variables $X, Y: \Omega \rightarrow \mathbb{R}$ and their corresponding copula $C:[0,1]^{2} \rightarrow[0,1]$ is defined in analogy to positive quadrant dependence by reversing the inequality signs in (5.8) or, equivalently, by $C \leq \Pi$.

Taking into account the duality between positive and negative quadrant dependence, the proof of the following result is analogous to the proof of Proposition 5.4.

Proposition 5.5. Let $C_{(a, b, c)}:[0,1]^{2} \rightarrow[0,1]$ be a polynomial copula of degree 5 , i.e., $(a, b, c) \in \mathbf{P C 5}$. The following are equivalent:

(i) The copula $C_{(a, b, c)}$ is negatively quadrant dependent.

(ii) The triplet $(a, b, c)$ is an element of the subset $\mathbf{P}_{\mathrm{NQD}}$ of $\mathbf{P C 5}$ given by

$$
\mathbf{P}_{\mathrm{NQD}}=\operatorname{Conv}(\{(0,0,0),(0,-1,0),(-1,0,0),(1,-1,-1),(1,0,-1),(0,1,-1),(-1,1,-1)\}) .
$$




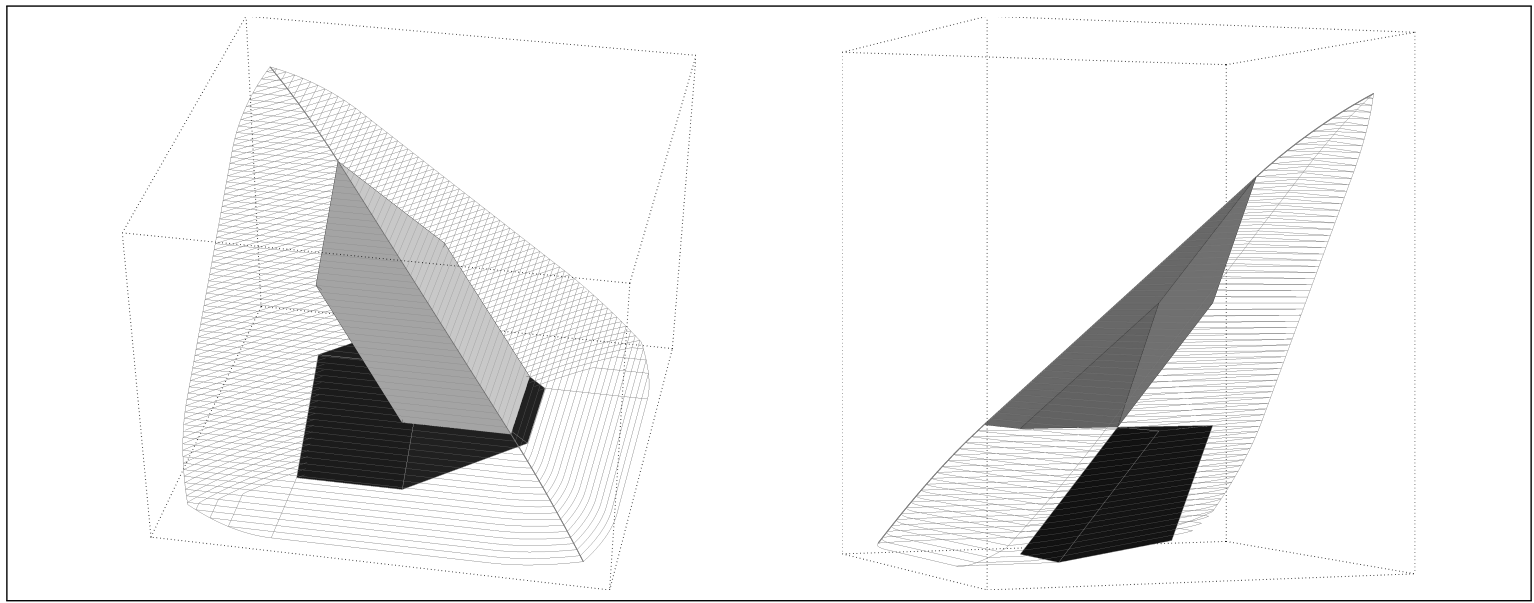

Figure 9: Parameter set of negatively (dark gray, bottom) and positively quadrant dependent polynomial copulas of degree 5 (light gray, top) from two different viewpoints (the set PC5 is indicated by the gray skeleton).

For each $c \in \mathbb{R}$ the level set $\mathbf{P}_{\mathrm{NQD}}{ }^{[c]}$ of the set of parameters $\mathbf{P}_{\mathrm{NQD}}$ of the set of all negatively quadrant dependent polynomial copulas of degree 5 is characterized as follows:

$$
\mathbf{P}_{\mathrm{NQD}}{ }^{[c]}= \begin{cases}\operatorname{Conv}(\{(-c,-1, c),(-c, 0, c),(0,-c, c),(-1,-c, c)\}) & \text { if } c \in[-1,0], \\ \emptyset & \text { otherwise. }\end{cases}
$$

The parameter sets $\mathbf{P}_{\mathrm{PQD}}$ and $\mathbf{P}_{\mathrm{NQD}}$ of positively and negatively quadrant dependent polynomial copulas of degree 5, respectively, are visualized in Figure 9. Obviously, we have $\mathbf{P}_{\mathrm{PQD}} \cap \mathbf{P}_{\mathrm{NQD}}=\{(0,0,0)\}$, i.e., the product copula $\Pi=C_{(0,0,0)}$ is the only (polynomial) copula (of degree 5 ) which is both negatively and positively quadrant dependent, as expected. Since each ultramodular copula is necessarily negatively quadrant dependent, we also have $\mathbf{P}_{\mathrm{UM}} \subseteq \mathbf{P}_{\mathrm{NQD}}$.

\subsection{Some automorphisms of polynomial copulas of degree five}

Given a copula $C:[0,1]^{2} \rightarrow[0,1]$, several other copulas are closely related to it, among them the $x$-flipping $C^{x f l i p}:[0,1]^{2} \rightarrow[0,1]$ of $C$ and the $y$-flipping $C^{y f l i p}:[0,1]^{2} \rightarrow[0,1]$ of $C$ (see [21]), and the survival copula $\widehat{C}:[0,1]^{2} \rightarrow[0,1]$ given by, respectively

$$
\begin{aligned}
& C^{\text {xflip }}(x, y)=y-C(1-x, y), \\
& C^{y f l i p}(x, y)=x-C(x, 1-y), \\
& \widehat{C}(x, y)=x+y-1+C(1-x, 1-y) .
\end{aligned}
$$

If $\mathcal{C}$ denotes the set of (bivariate) copulas, the mappings

$$
C \longmapsto C^{\text {xflip }}, \quad C \longmapsto C^{\text {yflip }}, \quad C \longmapsto \widehat{C}
$$

induce involutive bijections from $\mathcal{C}$ onto $\mathcal{C}$.

Obviously, each of the involutive bijections induced by (5.15) transforms a polynomial copula $C$ (of arbitrary degree $n$ ) into a polynomial copula $C$ of the same degree, i.e., the mappings in (5.15) induce also involutive bijections from PC5 onto PC5.

Since each polynomial copula $C$ of degree 5 can be identified with a parameter triplet $(a, b, c) \in \mathbf{P C} 5$, i.e., $C=C_{(a, b, c)}$ as defined in (2.13), we obtain the following formulas for the $x$-flipping, the $y$-flipping and the survival copula of a polynomial copula of degree 5 :

$$
\left(C_{(a, b, c)}\right)^{x f l i p}=C_{(a,-b,-a-c)}, \quad\left(C_{(a, b, c)}\right)^{y f l i p}=C_{(-a, b,-b-c)}, \quad \widehat{C_{(a, b, c)}}=C_{(-a,-b, a+b+c)},
$$


showing that the three mappings $C \longmapsto C^{x f l i p}, C \longmapsto C^{\text {fflip }}$ and $C \longmapsto \widehat{C}$ given in (5.15) induce involutive bijections from PC5 onto PC5.

From (5.16) it follows immediately which polynomial copulas of degree 5 are invariant under these automorphisms.

Corollary 5.6. Let $C_{(a, b, c)}:[0,1]^{2} \rightarrow[0,1]$ be a polynomial copula of degree 5 , i.e., $(a, b, c) \in \mathbf{P C 5}$.

(i) The copula $C_{(a, b, c)}$ is invariant under $x$-flipping, i.e., $\left(C_{(a, b, c)}\right)^{x f l i p}=C_{(a, b, c)}$, if and only if there is a parameter $\theta \in[-1,1]$ such that $(a, b, c)=(-2 \theta, 0, \theta)$.

(ii) The copula $C_{(a, b, c)}$ is invariant under $y$-flipping, i.e., $\left(C_{(a, b, c)}\right)^{y f l i p}=C_{(a, b, c)}$, if and only if there is a parameter $\theta \in[-1,1]$ such that $(a, b, c)=(0,-2 \theta, \theta)$.

(iii) The copula $C_{(a, b, c)}$ coincides with its survival copula, i.e., $\widehat{C_{(a, b, c)}}=C_{(a, b, c)}$, if and only if there is a parameter $\theta \in[-1,1]$ such that $(a, b, c)=(0,0, \theta)$, i.e., $C_{(a, b, c)}=C_{\theta}^{\mathrm{EFGM}}$.

Since the Eyraud-Farlie-Gumbel-Morgenstern copulas are polynomial copulas of degree 4, Corollary 5.6 (iii) tells us that no proper polynomial copula of degree 5 coincides with its survival copula. Therefore, polynomial copulas of degree 5 are more flexible than EFGM copulas since they do not induce radial symmetry.

Note also that, for bivariate copulas, the reflections considered in [28] (see also [50]) are exactly the $x$ flippings (5.12) and $y$-flippings (5.13) (which is no longer the case if one also considers $n$-dimensional copulas with $n>2$ ).

It is easy to see that the $x$-flipping and the $y$-flipping of a copula $C$ change its relationship to the product copula $\Pi$. Therefore, for each $(a, b, c) \in \mathbf{P C 5}$ we have (recall that $\Pi^{x f l i p}=\Pi^{\text {fflip }}=\Pi$ )

$$
\begin{aligned}
& C_{(a, b, c)} \leq \Pi \quad \Longleftrightarrow \quad\left(C_{(a, b, c)}\right)^{x f l i p} \geq \Pi \text { and }\left(C_{(a, b, c)}\right)^{y f l i p} \geq \Pi, \\
& C_{(a, b, c)} \geq \Pi \quad \Longleftrightarrow \quad\left(C_{(a, b, c)}\right)^{x f l i p} \leq \Pi \text { and }\left(C_{(a, b, c)}\right)^{y f l i p} \leq \Pi .
\end{aligned}
$$

As a consequence, the functions $C \longmapsto C^{x f l i p}$ and $C \longmapsto C^{\text {fflip }}$ in (5.15) can be seen as bijections between the sets of positively quadrant dependent, on the one hand, and negatively quadrant dependent, on the other hand, polynomial copulas of degree 5, while the construction of the survival copula $C \longmapsto \widehat{C}$ in (5.15) acts as an involution on both positively and negatively quadrant dependent polynomial copulas of degree 5 :

Corollary 5.7. Let $C_{(a, b, c)}:[0,1]^{2} \rightarrow[0,1]$ be a polynomial copula of degree 5 , i.e., $(a, b, c) \in \mathbf{P C 5}$. The following are equivalent:

(i) The copula $C_{(a, b, c)}$ is positively quadrant dependent.

(ii) The $x$-flipping $\left(C_{(a, b, c)}\right)^{x f l i p}$ of the copula $C_{(a, b, c)}$ is negatively quadrant dependent.

(iii) The $y$-flipping $\left(C_{(a, b, c)}\right)^{y f l i p}$ of the copula $C_{(a, b, c)}$ is negatively quadrant dependent.

(iv) The survival copula $\left(\widehat{C_{(a, b, c)}}\right)$ of the copula $C_{(a, b, c)}$ is positively quadrant dependent.

By duality, we obtain another set of four equivalent assertions if we replace in Corollary 5.7 consistently the property "positively quadrant dependent" by "negatively quadrant dependent" and vice versa.

We only mention that the $x$-flipping, the $y$-flipping and the construction of the survival copula of a polynomial copula $C_{(a, b, c)}$ of degree 5 map each extremal point of any of the convex parameter sets $\mathbf{P}_{\mathrm{PQD}}$ and $\mathbf{P}_{\mathrm{NQD}}$ to an extremal point of the respective range.

As already mentioned in Section 5.3, there is a special relationship between ultramodular copulas and stochastically decreasing copulas (see [66]).

If $(\Omega, \mathcal{A}, P)$ is a probability space, if $X, Y: \Omega \rightarrow \mathbb{R}$ are continuous random variables and if $C:[0,1]^{2} \rightarrow$ $[0,1]$ is the uniquely determined corresponding copula according to Theorem 2.1 then the copula $C$ is said to be stochastically increasing (SI for short) if for all $x, y \in \mathbb{R}$ the two functions $h_{(X>x, Y)}: \mathbb{R} \rightarrow[0,1]$ and $h_{(Y>y, X)}: \mathbb{R} \rightarrow[0,1]$ given by, respectively,

$$
h_{(X>x, Y)}(y)=P[X>x \mid Y=y] \quad \text { and } \quad h_{(Y>y, X)}(x)=P[Y>y \mid X=x]
$$


are monotone non-decreasing. Similarly, the copula $C$ is called stochastically decreasing (SD for short) if for all $x, y \in \mathbb{R}$ the two functions $h_{(X>x, Y)}: \mathbb{R} \rightarrow[0,1]$ and $h_{(Y>y, X)}: \mathbb{R} \rightarrow[0,1]$ given by (5.17) are monotone non-increasing.

Proposition 5.8. Let $C:[0,1]^{2} \rightarrow[0,1]$ be a copula. Then the following are equivalent:

(i) $C$ is stochastically decreasing.

(ii) Each horizontal and each vertical section of $C$ is convex.

(iii) $C^{x f l i p}$ is stochastically increasing.

(iv) Each horizontal and each vertical section of $C^{x f l i p}$ is concave.

(v) $C^{y f l i p}$ is stochastically increasing.

(vi) Each horizontal and each vertical section of $C^{y f l i p}$ is concave.

(vii) $C$ is ultramodular.

Proof. As a consequence of Theorem 5.2.9 and Corollary 5.2 in [66] we have (i) $\Longleftrightarrow$ (ii), (iii) $\Longleftrightarrow$ (iv) and (v) $\Longleftrightarrow$ (vi).

If for each $\left(x^{\star}, y^{\star}\right) \in[0,1]^{2}$ the horizontal section $C\left(\cdot, y^{\star}\right)$ and the vertical section $C\left(x^{\star}, \cdot\right)$ of a copula are convex then the horizontal section $C^{x f l i p}\left(\cdot, y^{\star}\right)$ given by $C^{x f l i p}\left(x, y^{\star}\right)=x-C\left(x, 1-y^{\star}\right)$ and the vertical section $C^{x f l i p}\left(x^{\star}, \cdot\right)$ given by $C^{x f l i p}\left(x^{\star}, y\right)=x^{\star}-C\left(x^{\star}, 1-y\right)$ are concave, showing that (ii) implies (iv). In a similar way the implication (iv) $\Longrightarrow$ (ii) and the equivalence (ii) $\Longleftrightarrow$ (vi) can be verified.

Finally, the equivalence (ii) $\Longleftrightarrow$ (vii) follows from [60, Corollary 4.1] and [49, Proposition 2.3].

Turning our attention to polynomial copulas of degree 5 , we are able to identify the parameter sets of stochastically decreasing and increasing polynomial copulas of degree 5 .

Corollary 5.9. Denote by $\mathbf{P}_{\mathrm{SD}} \subseteq \mathbf{P C} 5$ and $\mathbf{P}_{\mathrm{SI}} \subseteq \mathbf{P C} 5$ the parameter sets of all stochastically decreasing and increasing, respectively, polynomial copulas of degree 5. Then we have:

$$
\begin{gathered}
\mathbf{P}_{\mathrm{SD}}=\mathbf{P}_{\mathrm{UM}}, \\
\mathbf{P}_{\mathrm{SI}}=\operatorname{Conv}\left(\left\{(0,0,0),\left(-\frac{1}{3}, \frac{1}{3}, \frac{2}{3}\right),\left(-\frac{1}{2}, 0,1\right),\left(0, \frac{1}{2}, \frac{1}{2}\right),\left(\frac{1}{2}, \frac{1}{2}, \frac{1}{2}\right),\left(-\frac{1}{2},-\frac{1}{2}, \frac{3}{2}\right),\left(\frac{1}{2}, 0, \frac{1}{2}\right),\left(0,-\frac{1}{2}, 1\right),\left(\frac{1}{3},-\frac{1}{3}, \frac{2}{3}\right)\right\}\right) .
\end{gathered}
$$

Proof. The first equality is an immediate consequence of Propositions 5.3 and 5.8, and for the second equality it suffices to take into account Proposition 5.8 and (5.16).

\section{Dependence parameters of polynomial copulas of degree five and higher}

Given two random variables $X$ and $Y$, the full information about their dependence is contained in the corresponding copula (see Theorem 2.1). A number of dependence parameters (also called concordance measures) measure the degree of dependence of $X$ and $Y$ (or, equivalently, of $C$ ). In this paper we shall deal with Spearman's rho [91], Kendall's tau [46], Blomqvist's beta [10], and Gini's gamma [37]. 
Definition 6.1. Let $(\Omega, \mathcal{A}, P)$ be a probability space and let $X, Y: \Omega \rightarrow \mathbb{R}$ be continuous random variables with copula $C:[0,1]^{2} \rightarrow[0,1]$. The following dependence parameters are defined by

$$
\begin{aligned}
& \varrho_{X, Y}=\varrho_{C}=12 \iint_{[0,1]^{2}} C(x, y) d x d y-3, \\
& \tau_{X, Y}=\tau_{C}=4 \iint_{[0,1]^{2}} C(x, y) d C(x, y)-1, \quad \text { (Kendall's tau) } \\
& \beta_{X, Y}=\beta_{C}=4 C\left(\frac{1}{2}, \frac{1}{2}\right)-1, \\
& \gamma_{X, Y}=\gamma_{C}=4 \int_{0}^{1}(C(x, x)+C(x, 1-x)) d x-2 .
\end{aligned}
$$

The Fréchet-Hoeffding lower bound $W$, the product copula $\Pi$ and the Fréchet-Hoeffding upper bound $M$ given by (2.4) are characterized by the following properties of the dependence parameters considered in Definition 6.1:

$$
\varrho_{W}=\tau_{W}=\beta_{W}=\gamma_{W}=-1, \quad \varrho_{\Pi}=\tau_{\Pi}=\beta_{\Pi}=\gamma_{\Pi}=0, \quad \varrho_{M}=\tau_{M}=\beta_{M}=\gamma_{M}=1 .
$$

For the family of Eyraud-Farlie-Gumbel-Morgenstern copulas $\left(C_{\theta}^{\mathrm{EFGM}}\right)_{\theta \in[-1,1]}$ the corresponding dependence parameters (for more details see $[30,44,66]$ ) are given as follows:

$$
\varrho_{C_{\theta}^{\mathrm{EFGM}}}=\frac{\theta}{3}, \quad \tau_{C_{\theta}^{\mathrm{EFGM}}}=\frac{2 \theta}{9}, \quad \beta_{C_{\theta}^{\mathrm{EFGM}}}=\frac{\theta}{4}, \quad \gamma_{C_{\theta}^{\mathrm{EFGM}}}=\frac{4 \theta}{15} .
$$

As a consequence, for each $\theta \in[-1,1]$ we have

$$
\varrho_{C_{\theta}^{\mathrm{EFGM}}} \in\left[-\frac{1}{3}, \frac{1}{3}\right], \quad \tau_{C_{\theta}^{\mathrm{EFGM}}} \in\left[-\frac{2}{9}, \frac{2}{9}\right], \quad \beta_{C_{\theta}^{\mathrm{EFGM}}} \in\left[-\frac{1}{4}, \frac{1}{4}\right], \quad \gamma_{C_{\theta}^{\mathrm{EFGM}}} \in\left[-\frac{4}{15}, \frac{4}{15}\right] .
$$

If we switch to polynomial copulas of degree 5 , it is easy to show that the dependence parameters of $C_{(a, b, c)}$ can be computed in a simple way as functions of the parameters $a, b$ and $c$ :

Corollary 6.2. For each polynomial copula of degree 5, i.e., for each triplet $(a, b, c) \in \mathbf{P C 5}$, we have

(i) $\varrho_{C_{(a, b, c)}}=\frac{1}{6}(a+b+2 c)$;

(ii) $\tau_{C_{(a, b, c)}}=\frac{1}{9}(a+b+2 c)-\frac{1}{450} a b$;

(iii) $\beta_{C_{(a, b, c)}}=\frac{1}{8}(a+b+2 c)$;

(iv) $\gamma_{C_{(a, b, c)}}=\frac{2}{15}(a+b+2 c)$.

It is quite interesting that the values of the four dependence parameters considered here cover the same intervals for polynomial copulas of degree 5 as for polynomial copulas of degree 4, i.e., for EFGM copulas. Moreover, because of the symmetry of the parameter set PC5 we obtain the same intervals if we only consider the symmetric polynomial copulas of degree $5 C_{(a, a, c)}$, i.e., with $(a, a, c) \in \mathbf{P C} 5_{\text {sym }}$.

Proposition 6.3. For polynomial copulas of degree 5 the following equalities hold:

(i) $\left\{\varrho_{C_{(a, b, c)}} \mid(a, b, c) \in \mathbf{P C} 5\right\}=\left\{\varrho_{C_{(a, a, c)}} \mid(a, a, c) \in \mathbf{P C} 5_{\text {sym }}\right\}=\left[-\frac{1}{3}, \frac{1}{3}\right]$;

(ii) $\left\{\boldsymbol{\tau}_{C_{(a, b, c)}} \mid(a, b, c) \in \mathbf{P C} 5\right\}=\left\{\tau_{C_{(a, a, c)}} \mid(a, a, c) \in \mathbf{P C} 5_{\text {sym }}\right\}=\left[-\frac{2}{9}, \frac{2}{9}\right]$;

(iii) $\left\{\beta_{C_{(a, b, c)}} \mid(a, b, c) \in \mathbf{P C 5}\right\}=\left\{\beta_{C_{(a, a, c)}} \mid(a, a, c) \in \mathbf{P C 5}_{\text {sym }}\right\}=\left[-\frac{1}{4}, \frac{1}{4}\right]$;

(iv) $\left\{\gamma_{C_{(a, b, c)}} \mid(a, b, c) \in \mathbf{P C 5}\right\}=\left\{\gamma_{C_{(a, a, c)}} \mid(a, a, c) \in \mathbf{P C} 5_{\text {sym }}\right\}=\left[-\frac{4}{15}, \frac{4}{15}\right]$.

Proof. Note first that

$$
\{a+b+2 c \mid(a, b, c) \in \mathbf{P C} 5\}=\{2 a+2 c \mid(a, a, c) \in \mathbf{P C 5}\}=[-2,2]
$$

as a consequence of (4.6) and the fact that each EFGM copula $C_{\theta}^{\mathrm{EFGM}}=C_{(0,0, \theta)}$ with $\theta \in[-1,1]$ is a(n improper) polynomial copula of degree 5 . Therefore, the validity of (i), (iii) and (iv) follows immediately from Corollary 6.2 (i),(iii),(iv). 
In order to show that also (ii) holds, observe that because of (4.7), i.e., PC5 $\subset \mathbf{P}_{\left(B_{1}, B_{2}, B_{3}, B_{4}\right)}$, each parameter triplet $(a, b, c) \in \mathbf{P C 5}$ can be written as a convex combination of the coordinates of the points $B_{1}-B_{4}$. If we put $D_{\text {conv }}=\left\{\left(\alpha_{1}, \alpha_{2}, \alpha_{3}, \alpha_{4}\right) \in[0,1]^{4} \mid \alpha_{1}+\alpha_{2}+\alpha_{3}+\alpha_{4}=1\right\}$, then for each $(a, b, c) \in$ PC5 there is a quadruple $\left(\alpha_{1}, \alpha_{2}, \alpha_{3}, \alpha_{4}\right) \in D_{\text {conv }}$ such that

$$
(a, b, c)=\alpha_{1}(-2,-2,3)+\alpha_{2}(-2,2,-1)+\alpha_{3}(2,-2,-1)+\alpha_{4}(2,2,-1)
$$

or, equivalently,

$$
a=2\left(-\alpha_{1}-\alpha_{2}+\alpha_{3}+\alpha_{4}\right), \quad b=2\left(-\alpha_{1}+\alpha_{2}-\alpha_{3}+\alpha_{4}\right), \quad c=4 \alpha_{1}-1
$$

This implies

$$
\begin{aligned}
a+b+2 c & =4\left(\alpha_{1}+\alpha_{4}\right)-2, \\
a b & =4\left(\left(\alpha_{4}-\alpha_{1}\right)^{2}-\left(\alpha_{3}-\alpha_{2}\right)^{2}\right), \\
\frac{1}{9}(a+b+2 c)-\frac{1}{450} a b & =\frac{4}{9}\left(\alpha_{1}+\alpha_{4}\right)-\frac{2}{9}-\frac{2}{225}\left(\left(\alpha_{4}-\alpha_{1}\right)^{2}-\left(\alpha_{3}-\alpha_{2}\right)^{2}\right) .
\end{aligned}
$$

Now it is easy to see that the function $F: D_{\text {conv }} \rightarrow \mathbb{R}$ given by

$$
F\left(\alpha_{1}, \alpha_{2}, \alpha_{3}, \alpha_{4}\right)=\frac{4}{9}\left(\alpha_{1}+\alpha_{4}\right)-\frac{2}{9}-\frac{2}{225}\left(\left(\alpha_{4}-\alpha_{1}\right)^{2}-\left(\alpha_{3}-\alpha_{2}\right)^{2}\right)
$$

has no stationary point in $D_{\text {conv }}$ and, therefore, no relative maximum or minimum in the interior of $D_{\text {conv }}$. On the boundaries of $D_{\text {conv }}$, however, we find two extremal values of $F$ : the local minimum $-\frac{2}{9}$ for $\alpha_{1}=\alpha_{4}=0$ and $\alpha_{2}=\alpha_{3}=\frac{1}{2}$, and the local maximum $\frac{2}{9}$ for $\alpha_{1}=\alpha_{4}=\frac{1}{2}$ and $\alpha_{2}=\alpha_{3}=0$.

In other words, this means that the minimum of $F$ is attained for $(a, b, c)=(0,0,-1)$, and the maximum of $F$ for $(a, b, c)=(0,0,1)$. Therefore, we have $\tau_{C_{(0,0,-1)}}=\tau_{C_{-1}^{\mathrm{EFGM}}}=-\frac{2}{9}, \tau_{C_{(0,0,1)}}=\tau_{C_{1}^{\mathrm{EFGM}}}=\frac{2}{9}$, and $\tau_{C_{(a, b, c)}} \in$ ]$-\frac{2}{9}, \frac{2}{9}[$ for all $(a, b, c) \in \mathbf{P C} 5 \backslash\{(0,0,-1),(0,0,1)\}$.

Because of Corollary 6.2 there is a small difference between Kendall's tau and the other three dependence parameters. As pointed out in the proof of Proposition 6.3 (ii), the extremal values of Kendall's tau are only attained for a single polynomial copula of degree 5: $\tau_{C_{(0,0,-1)}}=-\frac{2}{9}$ and $\tau_{C_{(0,0,1)}}=\frac{2}{9}$. This is contrary to the situation of the other three dependence parameters considered here, where the extremal values of Spearman's rho $\left( \pm \frac{1}{3}\right)$, Blomqvist's beta $\left( \pm \frac{1}{4}\right)$, and Gini's gamma $\left( \pm \frac{4}{15}\right)$ are attained by all polynomial copulas of degree 5 of the form $C_{(a,-a,-1)}$ with $a \in[-1,1]$ and $C_{(1-c, 1-c, c)}$ with $c \in[0,2]$, respectively.

The results in this section allow us to formulate some preliminary observations concerning the so-called $\tau$ - $\varrho$-region of the set of polynomial copulas of degree 5 which describes the relationship between the dependence parameters $\tau$ and $\varrho$. Recall that for a set $\mathcal{S} \subseteq \mathcal{C}$ of copulas the $\tau$ - $\varrho$-region $\mathrm{R}_{\mathcal{S}}^{[\tau, \varrho]}$ is defined by $\mathrm{R}_{\mathcal{S}}^{[\tau, \varrho]}=\left\{\left(\tau_{C}, \varrho_{C}\right) \mid C \in \mathcal{S}\right\}$ (see Theorems 5.1.10, 5.1.11 and Corollary 5.1.12 in [66], and compare also [79]). Clearly, for the set of EFGM copulas we obtain a line segment: $\mathrm{R}_{\mathrm{EFGM}}^{[\tau, \rho]}=\left\{\left(t, \frac{3}{2} t\right) \mid t \in\left[-\frac{2}{9}, \frac{2}{9}\right]\right\}$.

From Corollary 6.2 (i)-(ii) and Proposition 6.3 (i)-(ii) it follows that the $\tau$ - $\varrho$-region $R_{\text {PC5 }}^{[\tau, \varrho]}$ of all polynomial copulas of degree 5 is a proper subset of the rectangle $\left[-\frac{2}{9}, \frac{2}{9}\right] \times\left[-\frac{1}{3}, \frac{1}{3}\right]$ (taking into account [66, Figure 5.4]) and a proper superset of $\mathrm{R}_{\mathrm{EFGM}}^{[\tau, \varrho]}$. To verify the latter, observe that for each $c \in[0,2]$ we have $(1-c, 1-c, c) \in$ PC5 and $\varrho_{C_{(1-c, 1-c, c)}}=\frac{1}{3}$. Because of $\{a b \mid a=b=1-c$ and $c \in[0,2]\}=[0,1]$ we obtain $\tau_{C_{(1-c, 1-c, c)}} \in$ $\left[\frac{2}{9}-\frac{1}{450}, \frac{2}{9}\right]$ for each $c \in[0,2]$, and therefore $\left[\frac{99}{450}, \frac{2}{9}\right] \times\left\{\frac{1}{3}\right\} \subseteq \mathrm{R}_{\mathbf{P C} 5}^{[\tau, \varrho]}$. The full characterization of the $\tau-\varrho-$ region $\mathrm{R}_{\mathbf{P C 5}}^{[\tau, \rho]}$ of polynomial copulas of degree 5 , however, will be the topic of future research.

Proposition 6.3 gives raise to the following question: what can be said about the values attained by the dependence parameters considered in this paper if all polynomial copulas (of any degree) as characterized by (2.9) are taken into account?

Proposition 6.4. Denote by $\mathrm{e}^{[\mathrm{poly}]}$ the set of all polynomial copulas as characterized by (2.9). Then we have:

$$
\left.\left\{\varrho_{C} \mid C \in \mathrm{e}^{[\text {poly }]}\right\}=\left\{\tau_{C} \mid C \in \mathrm{e}^{[\text {poly }]}\right\}=\left\{\beta_{C} \mid C \in \mathrm{e}^{[\text {poly }]}\right\}=\left\{\gamma_{C} \mid C \in \mathrm{e}^{[\text {poly }]}\right\}=\right]-1,1[.
$$


Proof. For each copula $C:[0,1]^{2} \rightarrow[0,1]$ also the function $D_{C}:[0,1]^{2} \rightarrow[0,1]$ given by (2.6) is a copula (see [53, Theorem 1] and Example 2.3 (ii)). Evidently, if $C$ is a polynomial copula of degree $k$ then $D_{C}$ is a

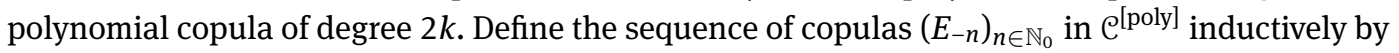

$$
E_{-n}= \begin{cases}\Pi & \text { if } n=0, \\ D_{E_{-(n-1)}} & \text { if } n \geq 1 .\end{cases}
$$

Then, following $[47,52]$, the sequence $\left(E_{-n}\right)_{n \in \mathbb{N}_{0}}$ converges to the Fréchet-Hoeffding lower bound $W$, i.e.,

$$
\lim _{n \rightarrow \infty} E_{-n}=W \text {. }
$$

Fix an arbitrary $r \in \mathbb{R} \backslash \mathbb{Z}$ with $r<0$, note that there is a unique $n_{r} \in \mathbb{N}$ such that $-n_{r}-1<r<-n_{r}$, and put

$$
E_{r}=-\left(r+n_{r}\right) E_{-\left(n_{r}+1\right)}+\left(r+n_{r}+1\right) E_{-n_{r}} .
$$

We see that $E_{r}$ is a convex combination of the two copulas $E_{-n_{r}}$ and $E_{-\left(n_{r}+1\right)}$, and thus also a copula. Then the parametric family $\left(E_{r}\right)_{r \in]-\infty, 0]}$ of copulas is continuous and monotone non-decreasing with respect to the parameter $r$. Taking into account (6.3) and the fact that $W$ and $\Pi$ are the only copulas where all the dependence parameters given in Definition 6.1 are equal to -1 and 0, respectively (see (6.1)), we obtain

$$
\left.\left.\left.\left.\left.\left.\left.\left.\left.\left.\left\{\varrho_{E_{r}} \mid r \in\right]-\infty, 0\right]\right\}=\left\{\tau_{E_{r}} \mid r \in\right]-\infty, 0\right]\right\}=\left\{\beta_{E_{r}} \mid r \in\right]-\infty, 0\right]\right\}=\left\{\gamma_{E_{r}} \mid r \in\right]-\infty, 0\right]\right\}=\right]-1,0\right] .
$$

To conclude the proof, it is enough to consider the $x$-flipping $C^{x f l i p}:[0,1]^{2} \rightarrow[0,1]$ of a copula $C$ [21] given by (5.12) which is also a copula. Note that for each copula $C$ we have $\varrho_{C \text { ffip }}=-\varrho_{C}, \tau_{C \times \text { flip }}=-\tau_{C}, \beta_{C \times f i p}=-\beta_{C}$, and $\gamma_{C^{x \text { fip }}}=-\gamma_{C}$. Moreover, if $C$ is a polynomial copula (of degree $k$ ) then also $C^{\text {xflip }}$ is a polynomial copula (of degree $k$ ).

Now define, for each real number $r \geq 0$, the copula $E_{r}:[0,1]^{2} \rightarrow[0,1]$ by $E_{r}=\left(E_{-r}\right)^{x f l i p}$. Noting $\Pi=\Pi^{x f l i p}$ and $M^{x f l i p}=W$, we see that the family of polynomial copulas $\left(E_{r}\right)_{r \in \mathbb{R}}$ attains all values from ] $-1,1$ [ for each of the dependence parameters given in Definition 6.1.

Since each copula $E_{r}$ is symmetric we even have shown the validity of the result of Proposition 6.4 if we restrict ourselves to symmetric copulas, i.e., to parameters in $(a, a, c) \in \mathbf{P C} 5_{\text {sym: }}$ :

Corollary 6.5. Denote by $\mathcal{C}_{\mathrm{sym}}^{[\mathrm{poly]}}$ the set of all symmetric polynomial copulas (of arbitrary degree). Then we have:

$$
\left.\left\{\varrho_{C} \mid C \in \mathcal{C}_{\text {sym }}^{[\text {poly] }}\right\}=\left\{\tau_{C} \mid C \in \mathcal{C}_{\text {sym }}^{[\text {poly] }}\right\}=\left\{\beta_{C} \mid C \in \mathcal{C}_{\text {sym }}^{[\text {poly }]}\right\}=\left\{\gamma_{C} \mid C \in \mathcal{C}_{\text {sym }}^{[\text {poly }]}\right\}=\right]-1,1[.
$$

\section{Concluding remarks}

Polynomial copulas can be seen as a genuine generalization of Eyraud-Farlie-Gumbel-Morgenstern copulas (which are, in fact, just polynomial copulas of degree 4). We have focused on polynomial copulas of degree 5 which necessarily have the form (2.13), i.e.,

$$
C_{(a, b, c)}(x, y)=x y+(a x+b y+c) x(1-x) y(1-y)
$$

for suitable parameters $(a, b, c) \in \mathbb{R}^{3}$. This parameter set PC5 $\subset \mathbb{R}^{3}$ was fully characterized and illustrated, giving analytical descriptions by means of inequalities of all level sets $\mathbf{P C}^{[\zeta]}=\{(a, b, c) \in \mathbf{P C} 5 \mid c=\zeta\}$, i.e., for each $\zeta \in[-1,1+2 \sqrt{2 / 3}]$ (see Tables 1-2).

Similarly, we have considered and characterized additional properties of polynomial copulas of degree 5 such as symmetry, Schur concavity, ultramodularity, and positive and negative quadrant dependence.

For each triplet $(a, b, c) \in \mathbf{P C} 5$ several dependence parameters (Spearman's rho, Kendall's tau, Blomqvist's beta and Gini's gamma) with respect to the corresponding polynomial copulas were computed. As can 
be seen from Corollary 6.2, here the expression $a+b+2 c$ plays a crucial role. As a consequence of (4.6), in PC5 this term $a+b+2 c$ attains all values in the interval [-2, 2], and it assumes the extremal values -2 and 2 for all parameters $(a,-a,-1)$ with $a \in[-1,1]$ and $(1-c, 1-c, c)$ with $c \in[0,2]$, respectively.

An interesting result is that Spearman's rho, Kendall's tau, Blomqvist's beta and Gini's gamma cover the same set of values for polynomial copulas of degree 5 as for polynomial copulas of degree 4, i.e., for EFGM copulas (Proposition 6.3). The situation changes if we consider polynomial copulas of arbitrary degree, in which case these four dependence parameters cover the maximal interval ]-1, 1[ (Proposition 6.4 and Corollary 6.5).

For polynomial copulas of higher degrees, the number of coefficients to be taken into account grows quadratically. For proper polynomial copulas of degree $n \geq 4$ we have to consider $\frac{(n-2)(n-3)}{2}$ coefficients. For example, if $n=6$ we have to deal with sixtuples $\left(a_{1}, a_{2}, \ldots, a_{6}\right) \in \mathbb{R}^{6}$, and all polynomial copulas of degree 6 necessarily have the form

$$
C(x, y)=x y+\left(a_{1} x^{2}+a_{2} x y+a_{3} y^{2}+a_{4} x+a_{5} y+a_{6}\right) x(1-x) y(1-y) .
$$

As an immediate consequence of Proposition 2.5 we must have $\frac{\partial^{2} C(x, y)}{\partial x \partial y} \geq 0$ which means that, e.g., for $(x, y)=$ $(0,0)$ we obtain $a_{6} \geq-1$ (compare this with the corresponding inequality $c \geq-1$ for polynomial copulas $C_{(a, b, c)}$ of degree 5 in Figure 1).

As mentioned in Example 2.3 (iii) (see [59]), the function $C:[0,1]^{2} \rightarrow[0,1]$ given by $(2.8)$ which can be rewritten as

$$
C(x, y)=x y+\frac{1}{2}(x y-x-y-1) x(1-x) y(1-y)
$$

is a polynomial copula of degree 6 (compare the results of [92] and (2.9)). It therefore corresponds to the 6-tuple $\left(0, \frac{1}{2}, 0,-\frac{1}{2},-\frac{1}{2},-\frac{1}{2}\right)$. However, no characterization of all 6-tuples $\left(a_{1}, a_{2}, \ldots, a_{6}\right) \in \mathbb{R}^{6}$ yielding a copula as given by (7.1) is known so far.

Acknowledgements: The authors are thankful for the support by the "Technologie-Transfer-Förderung" of the Upper Austrian Government (Wi-2014-200710/13-Kx/Kai). Also, the financial support for A. Šeliga, R. Mesiar and A. Kolesárová by the Slovak Research and Development Agency under the contract APVV-180052 and by the grants VEGA 1/0891/17, 1/0614/18 and VEGA 1/0006/19 is gratefully acknowledged. M. Kauers was supported by the Austrian Science Foundation (FWF) grants F5004 and P31571-N32. Open access funding was provided by the Slovak University of Technology, Bratislava.

The authors also appreciate the comments and suggestions of the two anonymous reviewers and of the journal editor during the reviewing process of this submission.

Wolfram Mathematica ${ }^{\circledR}$ (or simply Mathematica) is a technical computing system (featuring, among others, computer algebra, symbolic and numerical computations, and visualizations), developed by Wolfram Research of Champaign, Illinois (U.S.A., https://www.wolfram.com). In this paper we have used it mainly for the solution of polynomial inequalities, the (numerical) computation of integrals, and for the production of the figures.

\section{Appendix: Mathematica code}

Here we give some rather technical details of the functions which proved to be helpful when using Mathematica to identify the parameter triplets in PC5, i.e., to solve the polynomial inequality (3.2) subject to the linear constraints (4.6) as described in Subsection 4.1:

1. Define the polynomial appearing in (2.13).

poly $=1+c+2 a x-2 c x-3 a x^{-} 2+2 b y-2 c y-4 a x y$

$-4 b x y+4 c x y+6 a x^{\wedge} 2 y-3 b y^{\wedge} 2+6 b x y^{\wedge} 2$

2. Define a function for performing quantifier elimination as explained in Subsection 4.1. 


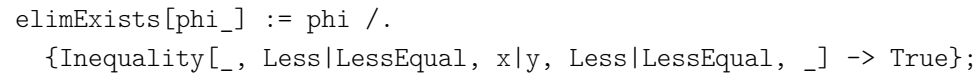

3. Define a function for simplifying formulas by replacing $u<x<v \vee v<x<w$ by $u<x<w$ and similar operations.

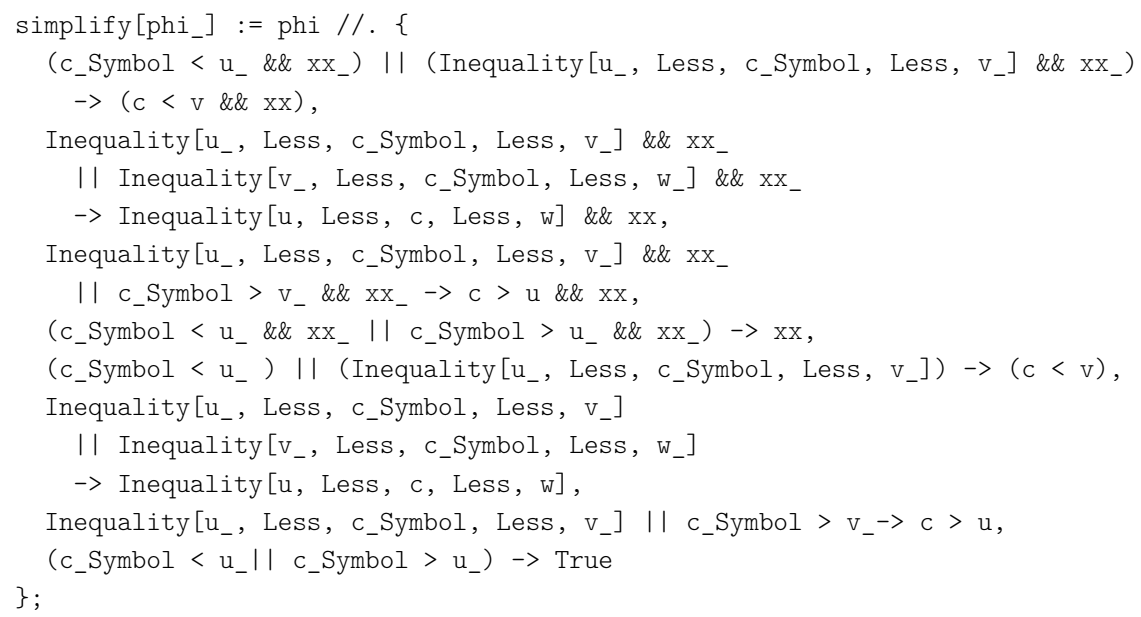

4. Define a function that performs the whole computation explained in Subsection 4.1, for a particular given variable order. The input argument should be a list of five variables such that the first three variables are $a, b, c$ (in any order) and the last two are $x, y$ (in any order). For any such input, the function returns a formula equivalent to the formula described in Subsection 4.2.

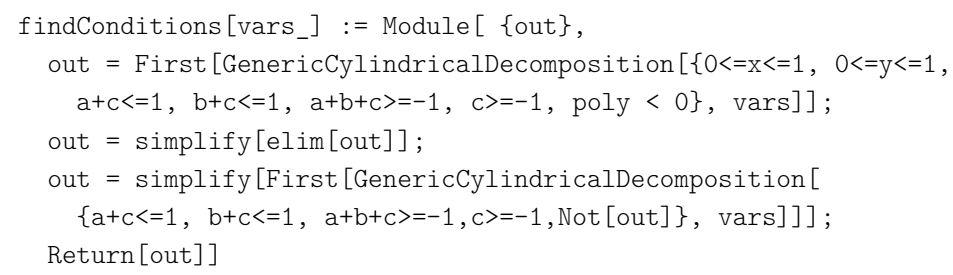

\section{References}

[1] Abel, N. H. (1826). Untersuchung der Functionen zweier unabhängig veränderlichen Größen $x$ und $y$, wie $f(x, y)$, welche die Eigenschaft haben, daß $f(z, f(x, y))$ eine symmetrische Function von $z, x$ und $y$ ist. J. Reine Angew. Math. 1, 11-15.

[2] Alsina, C., M. J. Frank, and B. Schweizer (2006). Associative Functions. World Scientific Publishing, Singapore.

[3] Amblard, C. and S. Girard (2009). A new extension of bivariate FGM copulas. Metrika 70, 1-17.

[4] Anakkamatee, W., S. Dhompongsa, and S. Tasena (2014). A constructive proof of the Sklar's theorem on copulas. J. Nonlinear Convex Anal. 15(6), 1137-1145.

[5] Barlow, R. E. and F. Proschan (1981). Statistical Theory of Reliability and Life Testing. To Begin With, Silver Spring MD.

[6] Basu, S., R. Pollack, and M.-F. Roy (2006). Algorithms in Real Algebraic Geometry. Second edition. Springer, Berlin.

[7] Bekrizadeh, H. and B. Jamshidi (2017). A new class of bivariate copulas: dependence measures and properties. Metron 75, 31-50.

[8] Bekrizadeh, H., G. A. Parham, and M. R. Zadkarmi (2012). The new generalization of Farlie-Gumbel-Morgenstern copulas. Appl. Math. Sci. 6(71), 3527-3533.

[9] Bernard, C. and A. Müller (2020). Dependence uncertainty bounds for the energy score and the multivariate Gini mean difference. Depend. Model. 8, 239-253.

[10] Blomqvist, N. (1950). On a measure of dependence between two random variables. Ann. Math. Statist. 21(4), 593-600.

[11] Boonmee, P. and S. Tasena (2020). Quadratic transformation of multivariate aggregation functions. Depend. Model. 8, 254-261.

[12] Brown, C. W. (2003). QEPCAD B: a program for computing with semi-algebraic sets using CADs. SIGSAM Bull. 37(4), 97-108.

[13] Cambanis, S. (1977). Some properties and generalizations of multivariate Eyraud-Gumbel-Morgenstern distributions. J. Multivariate Anal. 7(4), 551-559. 
[14] Cambanis, S. (1991). On Eyraud-Farlie-Gumbel-Morgenstern random processes. In G. Dall'Aglio, S. Kotz, and G. Salinetti (Eds.), Advances in Probability Distributions with Given Marginals, pp. 207-222. Kluwer Academic Publishers, Dordrecht.

[15] Caviness, B. F. and J. R. Johnson, editors (1998). Quantifier Elimination and Cylindrical Algebraic Decomposition. Springer, Wien.

[16] Collins, G. E. (1975). Quantifier elimination for real closed fields by cylindrical algebraic decompostion. In H. Brakhage (Ed.), Automata Theory and Formal Languages, pp. 134-183. Springer, Berlin.

[17] Cuadras, C. M. and W. Díaz (2012). Another generalization of the bivariate FGM distribution with two-dimensional extensions. Acta Comment. Univ. Tartu. Math. 16(1), 3-12.

[18] Dall'Aglio, G. (1956). Sugli estremi dei momenti delle funzioni di ripartizione doppia. Ann. Sc. Normale Super. Pisa Cl. Sci. (3) $10,35-74$.

[19] Dall'Aglio, G. (1959). Sulla compatibilità delle funzioni di ripartizione doppia. Rend. Mat. Appl. (3-4) 18, 385-413.

[20] Dall'Aglio, G. (1960). Les fonctions extrêmes de la classe de Fréchet à 3 dimensions. Publ. Inst. Statist. Univ. Paris 9 , 175-188.

[21] De Baets, B., H. De Meyer, J. Kalická, and R. Mesiar (2009). Flipping and cyclic shifting of binary aggregation functions. Fuzzy Set. Syst. 160(6), 752-765.

[22] Di Bernardino, E. and D. Rullière (2016). On an asymmetric extension of multivariate Archimedean copulas based on quadratic form. Depend. Model. 4, 328-347.

[23] Dolati, A. and M. Úbeda-Flores (2009). Constructing copulas by means of pairs of order statistics. Kybernetika 45(6), 992-1002.

[24] Drouet Mari, D. and S. Kotz (2001). Correlation and Dependence. Imperial College Press, London.

[25] Durante, F., J. Fernández-Sánchez, and C. Sempi (2012). Sklar’s theorem obtained via regularization techniques. Nonlinear Anal. 75(2), 769-774.

[26] Durante, F., J. Fernández-Sánchez, and C. Sempi (2013). A topological proof of Sklar’s theorem. Appl. Math. Lett. 26(9), 945-948.

[27] Durante, F., J. Fernández-Sánchez, and M. Úbeda-Flores (2013). Bivariate copulas generated by perturbations. Fuzzy Set. Syst. 228, 137-144.

[28] Durante, F. and S. Fuchs (2019). Reflection invariant copulas. Fuzzy Set. Syst. 354, 63-73.

[29] Durante, F. and C. Sempi (2003). Copulæ and Schur-concavity. Int. Math. J. 3(9), 893-905.

[30] Durante, F. and C. Sempi (2016). Principles of Copula Theory. CRC Press, Boca Raton FL.

[31] Eyraud, H. (1936). Les principes de la mesure des corrélations. Ann. Univ. Lyon, Sect. A 1, 30-47.

[32] Farlie, D. J. G. (1960). The performance of some correlation coefficients for a general bivariate distribution. Biometrika 47(3-4), 307-323.

[33] Faugeras, O. P. (2013). Sklar's theorem derived using probabilistic continuation and two consistency results. J. Multivariate Anal. 122, 271-277.

[34] Fernández-Sánchez, J. and M. Úbeda-Flores (2018). Proving Sklar’s theorem via Zorn’s lemma. Internat. J. Uncertain. Fuzz. 26(1), 81-85.

[35] Féron, R. (1956). Sur les tableaux de corrélation dont les marges sont données. Cas de l'espace à trois dimensions. Publ. Inst. Statist. Univ. Paris 5, 3-12.

[36] Fréchet, M. (1951). Sur les tableaux de corrélation dont les marges sont données. Ann. Univ. Lyon, Sect. A(3) $14,53-77$.

[37] Gini, C. (1955). Variabilità e mutabilità. In E. Pizetti and T. Salvemini (Eds.), Memorie di Metodologica Statistica. Libreria Eredi Virgilio Veschi, Roma.

[38] Gumbel, E. J. (1958). Distributions à plusieurs variables dont les marges sont données. C. R. Acad. Sci. Paris 246, 27172719.

[39] Hoeffding, W. (1940). Maßstabinvariante Korrelationstheorie. Schr. Math. Inst. Inst. Angew. Math. Univ. Berlin 5, 181-233. Also in Hoeffding, W. (1994). Scale-invariant correlation theory. In N. I. Fisher and P. K. Sen (Eds.), The Collected Works of Wassily Hoeffding, pp. 57-107. Springer, New York.

[40] Hoeffding, W. (1941). Maßstabinvariante Korrelationsmaße für diskontinuierliche Verteilungen. Arch. Math. Wirtsch.Sozialforschg. 7, 49-70. Also in Hoeffding, W. (1994). Scale-invariant correlation theory. In N. I. Fisher and P. K. Sen (Eds.), The Collected Works of Wassily Hoeffding, pp. 109-133. Springer, New York.

[41] Huang, J. S. and S. Kotz (1999). Modifications of the Farlie-Gumbel-Morgenstern distributions. A tough hill to climb. Metrika 49, 135-145.

[42] Hürlimann, W. (2017). A comprehensive extension of the FGM copula. Statist. Papers 58, 373-392.

[43] Joe, H. (1997). Multivariate Models and Dependence Concepts. Chapman \& Hall, London.

[44] Joe, H. (2015). Dependence Modeling with Copulas. CRC Press, Boca Raton FL.

[45] Kauers, M. (2011). How to use cylindrical algebraic decomposition. Sém. Lothar. Combin. 65, Article ID B65a, 16 pages.

[46] Kendall, M. G. (1938). A new measure of rank correlation. Biometrika 30(1-2), 81-93.

[47] Klement, E. P., A. Kolesárová, R. Mesiar, and S. Saminger-Platz (2017). On the role of ultramodularity and Schur concavity in the construction of binary copulas. J. Math. Inequal. 11(2), 361-381.

[48] Klement, E. P., M. Manzi, and R. Mesiar (2011). Ultramodular aggregation functions. Inform. Sci. 181(19), 4101-4111.

[49] Klement, E. P., M. Manzi, and R. Mesiar (2014). Ultramodularity and copulas. Rocky Mountain J. Math. 44(1), $189-202$. 
[50] Klement, E. P., R. Mesiar, and E. Pap (2002). Invariant copulas. Kybernetika 38(3), 275-286.

[51] Kolesárová, A. (2013). On quadratic constructions of copulas. In H. Bustince, J. Fernandez, R. Mesiar, and T. Calvo (Eds.), Aggregation Functions in Theory and in Practise, pp. 23-27. Springer, Berlin.

[52] Kolesárová, A., G. Mayor, and R. Mesiar (2015). Quadratic constructions of copulas. Inform. Sci. 310, 69-76.

[53] Kolesárová, A., R. Mesiar, and J. Kalická (2013). On a new construction of 1-Lipschitz aggregation functions, quasi-copulas and copulas. Fuzzy Set. Syst. 226, 19-31.

[54] Kolesárová, A. and R. Mesiar (2015). On linear and quadratic constructions of aggregation functions. Fuzzy Set. Syst. 268, 1-14.

[55] Komorník, J., M. Komorníková, and J. Kalická (2017). Dependence measures for perturbations of copulas. Fuzzy Set. Syst. 324, 100-116.

[56] Komorník, J., M. Komorníková, and J. Kalická (2018). Families of perturbation copulas generalizing the FGM family and their relations to dependence measures. In V. Torra, R. Mesiar, and B. De Baets (Eds.), Aggregation Functions in Theory and in Practice, pp. 53-63. Springer, Cham.

[57] Lehmann, E. L. (1966). Some concepts of dependence. Ann. Math. Statist. 37(5), 1137-1153.

[58] Ling, C.-H. (1965). Representation of associative functions. Publ. Math. Debrecen 12, 189-212.

[59] Manstavičius, M. and G. Bagdonas (2019). A class of bivariate copula mappings. Fuzzy Set. Syst. 354, 48-62.

[60] Marinacci, M. and L. Montrucchio (2005). Ultramodular functions. Math. Oper. Res. 30(2), 311-332.

[61] Marshall, A. W. and I. Olkin (1974). Majorization in multivariate distributions. Ann. Statist. 2(6), 1189-1200.

[62] Mesiar, R., M. Komorníková, and J. Komorník (2015). Perturbation of bivariate copulas. Fuzzy Set. Syst. 268, $127-140$.

[63] Mikami, T. (1997). Large deviations and central limit theorems for Eyraud-Farlie-Gumbel-Morgenstern processes. Statist. Probab. Lett. 35(1), 73-78.

[64] Morgenstern, D. (1956). Einfache Beispiele zweidimensionaler Verteilungen. Mitteilungsbl. Math. Statist. 8, 234-235.

[65] Nadarajah, S., E. Afuecheta, and S. Chan (2017). A compendium of copulas. Statistica 77(4), 279-328.

[66] Nelsen, R. B. (2006). An Introduction to Copulas. Second edition. Springer, New York.

[67] Nelsen, R. B., J. J. Quesada-Molina, and J. A. Rodríguez-Lallena (1997). Bivariate copulas with cubic sections. J. Nonparametr. Stat. 7(3), 205-220.

[68] Oertel, F. (2015). An analysis of the Rüschendorf transform - with a view towards Sklar's Theorem. Depend. Model. 3, 113-125.

[69] Puccetti, G. (2017). An algorithm to approximate the optimal expected inner product of two vectors with given marginals. J. Math. Anal. Appl. 451(1), 132-145.

[70] Puccetti, G. and R. Wang (2015). Extremal dependence concepts. Statist. Sci. 30(4), 485-517.

[71] Quesada-Molina, J. J. and J. A. Rodríguez-Lallena (1995). Bivariate copulas with quadratic sections. J. Nonparametr. Stat. 5(4), 323-337.

[72] Rodríguez-Lallena, J. A. and M. Úbeda-Flores (2008). Quasi-copulas with quadratic sections in one variable. Kybernetika 44(6), 879-890.

[73] Rodríguez-Lallena, J. A. and M. Úbeda-Flores (2010). Multivariate copulas with quadratic sections in one variable. Metrika 72, 331-349.

[74] Rovenţa, I. (2012). A note on Schur-concave functions. J. Inequal. Appl. 2012, no. 159, 9 pp.

[75] Rüschendorf, L. (1985). Construction of multivariate distributions with given marginals. Ann. Inst. Statist. Math. 37, 225233.

[76] Rüschendorf, L. (2009). On the distributional transform, Sklar's theorem, and the empirical copula process. J. Statist. Plann. Inference 139(11), 3921-3927.

[77] Saminger-Platz, S., A. Kolesárová, R. Mesiar, and E. P. Klement (2020). The key role of convexity in some copula constructions. Eur. J. Math. 6, 533-560.

[78] Saminger-Platz, S., A. Kolesárová, A. Šeliga, R. Mesiar, and E. P. Klement (2020). The impact on the properties of the EFGM copulas when extending this family. Fuzzy Set. Syst., to appear. Available at https://doi.org/10.1016/j.fss.2020.11.001.

[79] Schreyer, M., R. Paulin, and W. Trutschnig (2017). On the exact region determined by Kendall's $\tau$ and Spearman's $\varrho$. J. R. Stat. Soc. Ser. B. Stat. Methodol. 79(2), 613-633.

[80] Schur, I. (1923). Über eine Klasse von Mittelbildungen mit Anwendungen auf die Determinantentheorie. S. - B. Berlin. Math. Ges. 22, 9-20.

[81] Schwarz, G. (1985). Multivariate distributions with uniformly distributed projections. Ann. Probab. 13(4), 1371-1372.

[82] Schweizer, B. and A. Sklar (1961). Associative functions and statistical triangle inequalities. Publ. Math. Debrecen 8, 169-186.

[83] Schweizer, B. and A. Sklar (1963). Associative functions and abstract semigroups. Publ. Math. Debrecen 10, 69-81.

[84] Schweizer, B. and A. Sklar (1983). Probabilistic Metric Spaces. North-Holland, New York.

[85] Seidl, A. and T. Sturm (2003). A generic projection operator for partial cylindrical algebraic decomposition. Proceedings of the 2003 International Symposium on Symbolic and Algebraic Computation, pp. 240-247.

[86] Shaked, M. (1977). A family of concepts of dependence for bivariate distributions. J. Amer. Statist. Assoc. 72(359), 642-650.

[87] Shi, H.-N. (2007). Schur-convex functions related to Hadamard-type inequalities. J. Math. Inequal. 1(1), 127-136. 
[88] Shih, J.-H. and T. Emura (2018). Likelihood-based inference for bivariate latent failure time models with competing risks under the generalized FGM copula. Comput. Statist. 33, 1293-1323.

[89] Shih, J.-H. and T. Emura (2019). Bivariate dependence measures and bivariate competing risks models under the generalized FGM copula. Statist. Papers 60, 1101-1118.

[90] Sklar, A. (1959). Fonctions de répartition à $n$ dimensions et leurs marges. Publ. Inst. Statist. Univ. Paris 8, $229-231$.

[91] Spearman, C. (1904). The proof and measurement of association between two things. Am. J. Psychol. 15, 72-101.

[92] Sriboonchitta, S. and V. Kreinovich (2018). Why are FGM copulas successful? A simple explanation. Adv. Fuzzy Syst. 2018, Article ID 5872195, 5 pages.

[93] Strzeboński, A. Cylindrical Algebraic Decomposition. MathWorld-A Wolfram Web Resource. Available at http://mathworld. wolfram.com/CylindricalAlgebraicDecomposition.html.

[94] Strzeboński, A. (2000). Solving systems of strict polynomial inequalities. J. Symbolic Comput. 29(3), 471-480.

[95] Tarski, A. (1998). A decision method for elementary algebra and geometry. In B. F. Caviness and J. R. Johnson (Eds.), Quantifier Elimination and Cylindrical Algebraic Decomposition, pp. 24-84. Springer, Vienna. Reprinted from Tarski, A. (1951). A Decision Method for Elementary Algebra and Geometry. Second edition. University of California Press, Berkeley CA.

[96] Tasena, S. (2019). Characterization of quadratic aggregation functions. IEEE Trans. Fuzzy Systems 27(4), 824-829.

[97] Tasena, S. (2019). Polynomial copula transformations. Internat. J. Approx. Reason. 107, 65-78.

[98] Tchen, A. H. (1980). Inequalities for distributions with given marginals. Ann. Probab. 8(4), 814-827.

[99] Úbeda-Flores, M. (2008). Multivariate copulas with cubic sections in one variable. J. Nonparametr. Stat. 20(1), 91-98.

[100] Wisadwongsa, S. and S. Tasena (2018). Bivariate quadratic copula constructions. Internat. J. Approx. Reason. 92, 1-19. 